\title{
RENEWAL OF SINGULARITY SETS OF RANDOM SELF-SIMILAR MEASURES
}

\author{
JULIEN BARRAL, ${ }^{*}$ INRIA Rocquencourt \\ STÉPHANE SEURET, ${ }^{* *}$ Université Paris XII
}

\begin{abstract}
This paper investigates new properties concerning the multifractal structure of a class of random self-similar measures. These measures include the well-known Mandelbrot multiplicative cascades, sometimes called independent random cascades. We evaluate the scale at which the multifractal structure of these measures becomes discernible. The value of this scale is obtained through what we call the growth speed in Hölder singularity sets of a Borel measure. This growth speed yields new information on the multifractal behavior of the rescaled copies involved in the structure of statistically self-similar measures. Our results are useful in understanding the multifractal nature of various heterogeneous jump processes.
\end{abstract}

Keywords: Random measure; large deviations; Hausdorff dimension; self-similarity; fractal

2000 Mathematics Subject Classification: Primary 60G57; 60F10; 28A78

Secondary $28 \mathrm{~A} 80$

\section{Introduction}

This paper investigates new properties concerning the multifractal structure of random selfsimilar measures. The class of measures to which our results apply includes the well-known Mandelbrot multiplicative cascades [39], sometimes called independent random cascades. The case of another important class, the random Gibbs measures, was treated in [12]. Since these two important subclasses of random self-similar measures are extensively discussed in the sequel, in order to fix ideas we recall their definitions here.

- Random Gibbs measures, as considered in [12], are obtained as follows. Let $b \geq 2$ be an integer. Let $\phi$ be a Hölder continuous function on $\mathbb{R}^{d}$. Assume that $\phi$ is 1 -periodic in each variable. Also let $\omega=\left(\omega_{n}\right)_{n \geq 0}$ be a sequence of independent random phases uniformly distributed in $[0,1]^{d}$. Let $T$ be the shift transformation on $[0,1)^{d}$ :

$$
T\left(t_{1}, \ldots, t_{d}\right)=\left(b t_{1}(\bmod 1), \ldots, b t_{d}(\bmod 1)\right) .
$$

The Birkhoff sums associated with $\phi$ are defined by

$$
S_{n}(\phi, \omega)(t)=\sum_{k=0}^{n-1} \phi\left(T^{k} t+\omega_{k}\right) \quad \text { for all } t \in[0,1)^{d} \text { and all } n \geq 1,
$$

Received 3 March 2005; revision received 22 September 2006.

* Postal address: Equipe Sosso2, Domaine de Voluceau Rocquencourt, 78153 Le Chesnay cedex, France.

Email address: julien.barral@inria.fr

** Postal address: Laboratoire d'Analyse et de Mathématiques Appliquées, Faculté de Sciences et Technologie, Bat. P3, 4ème étage, 61 avenue du Général de Gaulle, 94010 Créteil cedex, France. Email address: seuret@univ-paris12.fr 
where $T^{k}$ stands for the $k$ th iterate of $T$. It follows from the thermodynamic formalism [48], [33] that the random sequence of measures on $[0,1]^{d}$ defined by

$$
\mu_{n}(\mathrm{~d} t)=\frac{\exp \left(S_{n}(\phi, \omega)(t)\right)}{\int_{[0,1]} \exp \left(S_{n}(\phi, \omega)(u)\right) \mathrm{d} u} \mathrm{~d} t
$$

converges almost surely, as $n \rightarrow \infty$, to a measure. This is called a random Gibbs measure.

- We also focus on independent random cascades (also referred to as canonical cascades [39]). Let $X$ be a real-valued random variable. Define $L: \mathbb{R} \ni q \mapsto d \log b+$ $\log \mathrm{E}(\exp (q X))$, and assume that $L(1)<\infty$. For every $b$-adic box $B$ included in $[0,1]^{d}$, let $X_{B}$ be a copy of $X$. Moreover, assume that the $X_{B}$ s are mutually independent. A branching random walk $S_{n}$ is then defined by

$$
S_{n}(t)=\sum_{1 \leq j \leq n} \sum_{B \in \mathcal{I}_{j}} \mathbf{1}_{B}(t) X_{B} \quad \text { for all } t \in[0,1)^{d} \text { and all } n \geq 1,
$$

where $\tau_{j}$ denotes the set of $b$-adic cubes of generation $j$.

The canonical cascade measure $\mu$ is then obtained as the almost-sure weak limit of the sequence $\mu_{n}$ on $[0,1]^{d}$ given by $\mu_{n}(\mathrm{~d} t)=\mathrm{E}(\exp X)^{-n} \exp \left(S_{n}(t)\right) \mathrm{d} t$. Let $\theta: \mathbb{R} \ni q \mapsto$ $(q L(1)-L(q)) / \log b$. In [39] and [31] it was shown that $\theta^{\prime}\left(1^{-}\right)>0$ is a necessary and sufficient condition for $\mu$ almost surely to be a positive measure with support equal to $[0,1]^{d}$.

We now discuss the purpose and main results of this work, and their connection with multifractal analysis. Multifractal analysis is a field introduced by physicists in the context of fully developed turbulence [24]. It is now widely accepted as a tool relevant in modeling other physical and social phenomena characterized by extreme spatial (or temporal) variability [40], [44], [35]. Let $B(t, r)$ denote the closed ball of radius $r$ centered at $t$. Given a positive measure $\mu$ defined on a compact subset of $\mathbb{R}^{d}$, performing the multifractal analysis of $\mu$ consists in computing (or estimating) the Hausdorff dimensions, $d_{\mu}(\alpha)$, of Hölder singularities sets $E_{\alpha}^{\mu}$. These sets $E_{\alpha}^{\mu}$ are the level sets associated with the Hölder exponent

$$
h_{\mu}(t)=\lim _{r \rightarrow 0^{+}} \frac{\log \mu(B(t, r))}{\log r}
$$

(whenever it is defined at $t$ ). Thus,

$$
E_{\alpha}^{\mu}=\left\{t: h_{\mu}(t)=\alpha\right\}
$$

Of course, these limit behaviors are incalculable numerically, both when simulating model measures and when processing real data. Nevertheless, this difficulty can be circumvented since the Hausdorff dimension of $E_{\alpha}^{\mu}$ can sometimes be numerically estimated by counting at scale $2^{-j}$ the number of dyadic boxes $B$ such that $\mu(B) \approx 2^{-j \alpha}$ (this could be done with any regular fine grid). This number can formally be defined, for any scale $j \geq 0$, any $\varepsilon>0$, and any $\alpha>0$, by

$$
N_{j}^{\varepsilon}(\alpha)=\operatorname{card}\left\{B \in \mathcal{I}_{j}: b^{-j(\alpha+\varepsilon)} \leq \mu(B) \leq b^{-j(\alpha-\varepsilon)}\right\}
$$


Then, when some formal multifractal conditions are fulfilled, it can be shown that, for some $\alpha>0$,

$$
d_{\mu}(\alpha)=\lim _{\varepsilon \rightarrow 0} \lim _{j \rightarrow \infty} \frac{\log N_{j}^{\varepsilon}(\alpha)}{\log 2^{j}} .
$$

This is the case, for instance, for the multifractal measures used as models [39] in the applications mentioned above. It is thus natural to seek theoretical results giving estimates of the first scale at which a substantial part of the singularity set $E_{\alpha}^{\mu}$ is discernible when measuring the $\mu$-mass of the elements $B$ of the regular grid. In other words, we search for the first generation $J \geq 0$ such that, for every $j \geq J, N_{j}^{\varepsilon}(\alpha) \approx 2^{j d_{\mu}(\alpha)}$. This is of course important for numerical applications and modeling.

The properties studied in this paper and in [12] rely on this problem. We provide new, accurate information on the fine structure of multiplicative cascades, thus providing some answers to the above problems. As an interesting by-product of this work, we find that Mandelbrot measures and Gibbs measures have very different behaviors from the statistical self-similarity point of view, although it is known that they cannot be distinguished by the form of their multifractal spectra. Finally, our results are critical tools for the Hausdorff dimension estimate of a new class of supremum limit sets (see (1.7)) involved in multifractal analysis of recently studied jump processes [10], [9], [11].

\subsection{A definition of random self-similar measures}

We now specify what we mean by a random self-similar measure. Our point of view takes into account a structure which often arises in the construction of random measures generated by multiplicative processes.

Let $\mathcal{I}$ be the set of closed $b$-adic subhypercubes of $[0,1]^{d}$. A random measure $\mu(\omega)$ on $[0,1]^{d}, d \geq 1$, is said to be random self-similar if there exist an integer $b \geq 2$, a sequence $\left(Q_{n}(t, \omega)\right)_{n \geq 1}$ of random nonnegative functions, and a sequence of random measures $\left(\mu^{I}\right)_{I \in \mathfrak{I}}$ on $[0,1]^{d}$ such that

1. for every $I \in \mathcal{I}$ and $g \in C\left([0,1]^{d}, \mathbb{R}\right.$ ) (the space of real-valued, continuous functions on $\left.[0, d]^{d}\right)$,

$$
\int_{[0,1]^{d}} g(u) \mu(\omega)(\mathrm{d} u) \stackrel{\mathrm{D}}{=} \int_{[0,1]^{d}} g(u) \mu^{I}(\omega)(\mathrm{d} u)
$$

(where 'D ' denotes equality in distribution), and

2. with probability 1 , for every $n \geq 1, I \in \mathcal{I}$ of generation $n$, and $g \in C(I, \mathbb{R})$,

$$
\int_{I} g(v) \mu(\omega)(\mathrm{d} v)=\ell(I) \int_{I} Q_{n}(v, \omega) g(v) \mu^{I}(\omega) \circ f_{I}^{-1}(\mathrm{~d} v),
$$

where $f_{I}$ stands for a similitude that maps $[0,1]^{d}$ onto $I$ and $\ell$ is the Lebesgue measure.

Property 1 asserts that the measures $\mu^{I}$ and $\mu$ have the same probability distribution. Property 2 asserts that, up to the density $Q_{n}$, the behavior of the restriction of $\mu$ to $I$ is governed by the rescaled copy $\mu^{I}$ of $\mu$. Of course, the random density $Q_{n}(t, \cdot)$ plays a fundamental role, both in the construction of the measure $\mu$, which is often equal to the almost-sure weak limit of $Q_{n}(t, \cdot) \ell$, and in the local behavior of $\mu$ at scale $b^{-n}$.

We restrict ourselves to measures with support equal to $[0,1]^{d}$. Up to technical refinements, our point of view can easily be extended to measures whose support is the limit set of more general iterated random similitude systems (see [27], [21], [45], [1], and [5]). 
The classes of measures described above illustrate conditions (1.4) and (1.5). Gibbs measures appear in random dynamical systems [33]. Independent multiplicative cascades [39], [31] are in fact contained in a wider class of $[0,1]^{d}$-martingales (in the sense of [29] and [30]); see [7] for details. This larger class also contains compound Poisson cascades [6] and their extensions [3], [7]. As claimed above, these two classes are quite similar regarding their multifractal structure, in the sense that any measure from these classes is governed by the socalled multifractal formalism [17], [46]. However, the study of their self-similarity properties reveals the notable differences between them. These differences are consequences of their construction schemes: for the class of random Gibbs measures, the copies $\mu^{I}$ used in (1.4) and (1.5) only depend on the generation of $I$, while, as illustrated by the construction of canonical cascades, they are all different for $[0,1]^{d}$-martingales (since they depend on the interval $I$ ).

This difference is quantitatively measured using a concept which is related to the multifractal structure, namely the growth speed in the $\mu^{I}$ Hölder singularity sets $E_{\alpha}^{\mu^{I}}$ (see Section 1.3 and Theorems 1.1 and 1.2 in Section 1.4, below). For independent random cascades, this quantity is precisely defined and studied in the rest of the paper. It yields an estimate of the largest scale at which the observation of the mass distribution of the $\mu^{I}$ accurately coincides with the prediction of the multifractal formalism.

\subsection{New supremum limit sets and conditioned ubiquity}

The notion of growth speed in Hölder singularity sets (defined in Section 1.3) naturally appears in the computation of the Hausdorff dimension of a new type of supremum limit set. These supremum limit sets are closely connected to the level sets of the pointwise Hölder exponent of some heterogeneous jump processes considered in [10] and [11]. This is why these sets are so interesting.

Let $\mu$ be a finite Borel measure whose support is $[0,1]$. The heterogeneous jump processes we consider are either purely discontinuous measures which have the form

$$
\sum_{j \geq 0} \sum_{0 \leq k \leq b^{j}-1} j^{-2} \mu\left(\left[k b^{-j},(k+1) b^{-j}\right]\right) \delta_{k b^{-j}},
$$

or the Lévy processes $X$ in multifractal time $\mu$ defined as $(X \circ \mu([0, t]))_{0 \leq t \leq 1}$. The fractal geometry of supremum limit sets already occupies an important role in determining the multifractal nature of homogeneous sums of Dirac masses [22] and Lévy processes [28]. In this homogeneous context, the measure $\mu$ is the Lebesgue measure, and computing the dimension of these sets relies on the notion of ubiquity (see [19], for instance). The study of $(X \circ \mu([0, t]))_{0 \leq t \leq 1}$ requires the notion of heterogeneous (or conditioned) ubiquity introduced in [10] and [8]. The work in [11] is a fundamental step in the study of the fractal nature of the path of processes under multifractal subordination. The importance of this topic comes from the fact that such processes have been introduced as relevant models in applications, especially in mathematical finance [40], [42], [41]. The reader will find an extensive study of processes in multifractal time in [50], which provides, for instance, the large deviation spectrum of Lévy processes in multifractal time, that is, a statistical description of the variations of these processes, rather than the geometric one given in [11].

We now describe the nature of the supremum limit sets discussed above. Let $\mu$ be a random self-similar measure whose support is $[0,1]$. Let $\tilde{x}=\left(x_{n}\right)_{n \geq 1}$ denote a countable sequence of points in $[0,1]^{d}$, and let $\tilde{\lambda}=\left(\lambda_{n}\right)_{n \geq 1}$ be a positive sequence decreasing to 0 such that $\lim \sup _{n \rightarrow \infty} B\left(x_{n}, \lambda_{n}\right)=[0,1]$. For $h>0, \xi>1$, and $\tilde{\varepsilon}=\left(\varepsilon_{n}\right)_{n \geq 1}$, the latter a positive 
sequence converging to 0 , let

$$
K(\mu, h, \xi, \tilde{x}, \tilde{\lambda}, \tilde{\varepsilon})=\bigcap_{N \geq 1} \bigcup_{\left\{n \geq N: \lambda_{n}^{h+\varepsilon_{n}} \leq \mu\left(\left[x_{n}-\lambda_{n}, x_{n}+\lambda_{n}\right]\right) \leq \lambda_{n}^{h-\varepsilon_{n}}\right\}}\left[x_{n}-\lambda_{n}^{\xi}, x_{n}+\lambda_{n}^{\xi}\right]
$$

Heuristically, $K(\mu, h, \xi, \tilde{x}, \tilde{\lambda}, \tilde{\varepsilon})$ contains the points that infinitely often belong to an interval of the form $\left[x_{n}-\lambda_{n}^{\xi}, x_{n}+\lambda_{n}^{\xi}\right]$, upon the condition that $\mu\left(\left[x_{n}-\lambda_{n}, x_{n}+\lambda_{n}\right]\right) \sim \lambda_{n}^{h}$. This condition implies that $\mu$ has roughly Hölder exponent $h$ at $x_{n}$ at scale $\lambda_{n}$. One of the main results of [10], [8], and [9] is the computation of the Hausdorff dimension of $K(\mu, h, \xi, \tilde{x}, \tilde{\lambda}, \tilde{\varepsilon})$. The value of this dimension is related to the free energy function $\tau_{\mu}$ considered in the multifractal formalism for measures in [26] and [17]. For every $q \in \mathbb{R}$ and for every integer $n \geq 1$, introduce the quantities

$$
\tau_{\mu, n}(q)=-\frac{1}{n} \log _{b}\left(\sum_{I \in \mathcal{I}_{n}} \mu(I)^{q}\right) \quad \text { and } \quad \tau_{\mu}(q)=\liminf _{n \rightarrow \infty}\left(\tau_{\mu, n}(q)\right) .
$$

The Legendre transform, $\tau_{\mu}^{*}$, of $\tau_{\mu}$ at $h>0$ is defined as $\tau_{\mu}^{*}(h):=\inf _{q \in \mathbb{R}}\left(h q-\tau_{\mu}(q)\right)$.

Under suitable assumptions, the authors proved in [10] and [8] that, for all $h$ such that $\tau_{\mu}^{*}(h)>0$ and all $\xi \geq 1$, there exists an $\tilde{\varepsilon}$ such that, with probability 1 ,

$$
\operatorname{dim}(K(\mu, h, \xi, \tilde{x}, \tilde{\lambda}, \tilde{\varepsilon}))=\tau_{\mu}^{*}(h) / \xi
$$

(where $\operatorname{dim}(\cdot)$ stands for Hausdorff dimension).

This result is a nontrivial generalization of what are referred to as 'ubiquity properties' (see [19] and references therein) of the resonant system $\left\{\left(x_{n}, \lambda_{n}\right)\right\}_{n}$ (see [2]). The main difficulty here lies in the fact that $\mu$ may be a multifractal measure and not just the uniform Lebesgue measure. Results on growth speed in Hölder singularity sets are necessary to obtain estimate (1.7).

Suppose that $\mu$ is an independent random cascade whose support is $[0,1]$, and that $X$ is a stable Lévy process of index $\beta \in(0,1)$. Let $v$ denote the derivative of $(X \circ \mu([0, t]))_{0 \leq t \leq 1}$. It was shown in [11] that there exist $\tilde{x}, \tilde{\lambda}$, and $\tilde{\varepsilon}$ as above such that, for every $h \in\left(0, \tau_{\mu}^{\prime}(1) / \beta\right.$ ], the level set $E_{h}^{v}$ differs from $K\left(\mu, \tau_{\mu}^{\prime}(1), \tau_{\mu}^{\prime}(1) / \beta h, \tilde{x}, \tilde{\lambda}, \tilde{\varepsilon}\right)$ by (roughly speaking) only a small set.

\subsection{Growth speed in the Hölder singularity sets of $\mu^{I}$}

Let $\mu$ be a random self-similar, positive Borel measure as described in Section 1.1. As we said, multifractal analysis of $\mu$ [27], [34], [43], [23], [1], [14], [5] usually considers Hölder singularities sets of the form (1.1) and their Hausdorff dimension $d_{\mu}(\alpha)$, which is a measure of their size. The method used to compute $d_{\mu}(\alpha)$ is to find a random measure $\mu_{\alpha}$ (of the same nature as $\mu$ ) that is concentrated on $E_{\alpha}^{\mu} \cap E_{\tau_{\mu}^{*}(\alpha)}^{\mu_{\alpha}}$. This measure is often referred to as an analyzing measure of $\mu$ at $\alpha$. Then, by the Billingsley lemma ([16, pp. 136-145]), we obtain $d_{\mu}(\alpha)=\tau_{\mu}^{*}(\alpha)$, and the multifractal formalism for measures developed in [17] is said to hold for $\mu$ at $\alpha$. Finally, the estimate (1.3) is a direct consequence of the multifractal formalism [49] for the large deviation spectrum. Thus, the existence of $\mu_{\alpha}$ has important consequences regarding the possibility of measuring the mass distribution of $\mu$ at high enough resolutions. 
In this paper we refine the classical approach by considering, instead of the level sets $E_{\alpha}^{\mu}$, the finer level sets $\tilde{E}_{\alpha, p}^{\mu}$ and $\tilde{E}_{\alpha}^{\mu}$ defined, for a sequence $\left(\varepsilon_{n}\right)_{n}$ decreasing to 0 , by

$$
\begin{aligned}
\tilde{E}_{\alpha, p}^{\mu} & =\left\{t \in[0,1]^{d}: b^{-n\left(\alpha+\varepsilon_{n}\right)} \leq \mu\left(I_{n}(t)\right) \leq b^{-n\left(\alpha-\varepsilon_{n}\right)} \text { for all } n \geq p\right\} \\
\tilde{E}_{\alpha}^{\mu} & =\bigcup_{p \geq 1} E_{\alpha, p}^{\mu},
\end{aligned}
$$

where $I_{n}(t)$ denotes the $b$-adic cube of generation $n$ containing $t$. It is possible to choose $\left(\varepsilon_{n}\right)_{n \geq 1}$ such that, for all the exponents $\alpha$ for which $\tau_{\mu}^{*}(\alpha)>0$, with probability 1 we have $\mu_{\alpha}\left(\tilde{E}_{\alpha}^{\mu}\right)=\left\|\mu_{\alpha}\right\|$, where $\left\|\mu_{\alpha}\right\|$ denotes the total mass of $\mu_{\alpha}$.

Since the set sequence $\left(\tilde{E}_{\alpha, p}^{\mu}\right)_{p \geq 1}$ is nondecreasing and $\mu_{\alpha}\left(\tilde{E}_{\alpha}^{\mu}\right)=\left\|\mu_{\alpha}\right\|$, we can define the growth speed of $\left(\tilde{E}_{\alpha, p}^{\mu}\right)$ as the smallest value of $p$ for which the $\mu_{\alpha}$-measure of $\tilde{E}_{\alpha, p}^{\mu}$ reaches a certain positive fraction $f \in(0,1)$ of the mass of $\mu_{\alpha}$, i.e.

$$
G S(\mu, \alpha)=\inf \left\{p: \mu_{\alpha}\left(\tilde{E}_{\alpha, p}^{\mu}\right) \geq f\left\|\mu_{\alpha}\right\|\right\}
$$

For each copy $\mu^{I}$ of $\mu$, the corresponding family of analyzing measures $\mu_{\alpha}^{I}$ exists and is related to $\mu^{I}$ as $\mu_{\alpha}$ is related to $\mu$. The result we focus on in the following is the asymptotic behavior (as the generation of $I$ goes to $\infty$ ) of

$$
G S\left(\mu^{I}, \alpha\right)=\inf \left\{p: \mu_{\alpha}^{I}\left(\tilde{E}_{\alpha, p}^{\mu^{I}}\right) \geq f\left\|\mu_{\alpha}^{I}\right\|\right\} .
$$

This number yields an estimate of the number of generations needed to observe a substantial amount of the singularity set $E_{\alpha}^{\mu^{I}}$. Let

$$
\mathcal{N}_{n}\left(\mu^{I}, \alpha\right)=\operatorname{card}\left\{B \in \mathcal{I}_{n}: b^{-n\left(\alpha+\varepsilon_{n}\right)} \leq \mu^{I}(B) \leq b^{-n\left(\alpha-\varepsilon_{n}\right)}\right\} .
$$

As a counterpart to controlling $G S\left(\mu^{I}, \alpha\right)$, we shall also control the smallest rank $n$ for which $\mathcal{N}_{n}\left(\mu^{I}, \alpha\right)$ behaves like $b^{n \tau_{\mu}^{*}(\alpha)}$. This rank is defined using

$$
G S^{\prime}\left(\mu^{I}, \alpha\right)=\inf \left\{p: b^{n\left(\tau_{\mu}^{*}(\alpha)-\varepsilon_{n}\right)} \leq \mathcal{N}_{n}\left(\mu^{I}, \alpha\right) \leq b^{n\left(\tau_{\mu}^{*}(\alpha)+\varepsilon_{n}\right)} \text { for all } n \geq p\right\}
$$

and yields far more precise information than a result like (1.3). We should expect that $\left(\varepsilon_{n}\right)$ and $G S\left(\mu^{I}, \alpha\right)$ are related through some constraints. This is indeed the case and this point is discussed in Theorem 3.2 and Remarks 3.1 and 3.2.

\subsection{A simplified version of the main results}

In this paper we focus on the one-dimensional case and we deal with independent random cascades, which are a slight extension of the first example of [0,1]-martingales introduced in [39] (see Section 3.1). We start by recalling the theorem proved in [12], and then give simplified versions of the main results, detailed in Section 3.

Theorem 1.1. Let $\mu$ be a random Gibbs measure as defined above (in particular, in (1.4) and (1.5), $\mu^{I}=\mu^{I^{\prime}}$ if $I$ and $I^{\prime}$ are of the same generation). Let $\beta>0$. There exists a choice of $\left(\varepsilon_{n}\right)_{n \geq 1}$ such that, with probability 1 , for all $\alpha>0$ such that $\tau_{\mu}^{*}(\alpha)>0$, if I is of large enough generation $j$ then $G S\left(\mu^{I}, \alpha\right) \leq \exp (\sqrt{\beta \log j})$.

The fact that $G S\left(\mu^{I}, \alpha\right)$ behaves like $o(j)$ as $j \rightarrow \infty$ is a crucial property needed to establish (1.7) for random Gibbs measures.

Under suitable assumptions, we have the following result (see Theorem 3.2), which shall be compared with Theorem 1.1. 
Theorem 1.2. Let $\mu$ be an independent random cascade. Let $\eta>0$. There exists a choice of $\left(\varepsilon_{n}\right)_{n \geq 1}$ such that, with probability 1 , for all $\alpha>0$ such that $\tau_{\mu}^{*}(\alpha)>0$, if I is of large enough generation $j$ then $G S\left(\mu^{I}, \alpha\right) \leq j \log ^{\eta} j$.

Consequently, we lose the uniform upper bound of $o(j)$ for $G S\left(\mu^{I}, \alpha\right)$ when $I \in \mathcal{I}_{j}$. In fact this 'worse' behavior is not surprising. Indeed, for an independent random cascade, at each resolution $j$ the behaviors at small scales of $b^{j}$ distinct measures $\mu^{I}$ have to be controlled simultaneously. However, this technical difficulty can be circumvented by using a refinement of Theorem 1.2 (see Theorems 3.3 and 5.1), which is enough to prove (1.7).

Theorem 1.3. Let $\mu$ be an independent random cascade. Let $\eta>0$. There exists a choice of $\left(\varepsilon_{n}\right)_{n \geq 1}$ such that, with probability 1 , for every $\alpha>0$ such that $\tau_{\mu}^{*}(\alpha)>0, \mu$-almost every $t$, and large enough $j, G S\left(\mu^{B_{j}(t)}, \alpha\right) \leq j \log ^{-\eta} j$ (where $B_{j}(t)$ denotes the $b$-adic box of generation $j$ containing $t$ ).

The paper is organized as follows. Section 2 gives new definitions and establishes two general propositions useful for our main results. In Section 3 independent random cascades are defined in an abstract way. This makes it possible to consider Mandelbrot measures, as well as their substitute in the critical case of degeneracy. Then the main results (Theorems 3.1, 3.2, and 3.3) are stated and proved. Theorem 3.4 is a counterpart to Theorem 1.2 in terms of $G S^{\prime}\left(\mu^{I}, \alpha\right)$ (recall (1.11)). Theorem 3.5 concerns a problem connected with the estimate of the growth speed in singularity sets, namely the estimation of the speed of convergence of $\tau_{\mu, n}$ to $\tau_{\mu}$. Section 4 provides the proofs of the results stated in Section 3. Finally, Section 5 is devoted to the version of Theorem 3.3 needed to recover (1.7).

The techniques presented in this paper can be applied to derive similar results for other random self-similar [0, 1]-martingales described in [6], [3], and [7].

\section{General estimates for the growth speed in singularity sets}

\subsection{Measure of fine-level sets: a neighboring boxes condition}

Let $(\Omega, \mathcal{B}, \mathrm{P})$ stand for the probability space on which the random variables in this paper are defined. Fix an integer $b \geq 2$ and let $\mathcal{A}=\{0, \ldots, b-1\}$. For every $w \in \mathcal{A}^{*}=\bigcup_{n \geq 0} \mathcal{A}^{n}$ $\left(\mathcal{A}^{0}:=\{\varnothing\}\right)$, let $I_{w}$ be the closed $b$-adic subinterval of $[0,1]$ naturally encoded by $w$. If $w \in \mathcal{A}^{n}$, we set $|w|=n$. For $n \geq 1$ and $0 \leq k \leq b^{n}-1$, we denote the interval $\left[k b^{-n},(k+1) b^{-n}\right)$ by $I_{n, k}$. If $t \in[0,1)$ then $k_{n, t}$ is the unique integer such that $t \in\left[k_{n, t} b^{-n},\left(k_{n, t}+1\right) b^{-n}\right)$. We denote by $w^{(n)}(t)$ the unique element $w$ of $\mathcal{A}^{n}$ such that $I_{w}=\left[k_{n, t} b^{-n},\left(k_{n, t}+1\right) b^{-n}\right]$. With $w \in \mathcal{A}^{n}$ we can associate a unique number, $i(w) \in\left\{0,1, \ldots, b^{n}-1\right\}$, such that $I_{w}=$ $\left[i(w) b^{-n},(i(w)+1) b^{-n}\right]$. Then, for $v, w \in \mathcal{A}^{n}, \delta(v, w)$ stands for $|i(v)-i(w)|$. Let $\mu$ and $m$ be two positive Borel measures with supports equal to [0,1], let $\tilde{\varepsilon}=\left(\varepsilon_{n}\right)_{n \geq 0}$ be a positive sequence, let $N \geq 1$, and let $\alpha \geq 0$.

We consider a slight refinement of the sets introduced in (1.8) and (1.9). For $p \geq 1$, we define

$$
\begin{aligned}
& E_{\alpha, p}^{\mu}(N, \tilde{\varepsilon})=\left\{t \in[0,1]: b^{\gamma n\left(\alpha-\gamma \varepsilon_{n}\right)} \mu\left(I_{w}\right)^{\gamma} \leq 1 \text { for all } n \geq p,\right. \\
& \left.\qquad \text { all } w \in \mathcal{A}^{n} \text { such that } \delta\left(w, w^{(n)}(t)\right) \leq N, \text { and all } \gamma \in\{-1,1\}\right\}, \\
& E_{\alpha}^{\mu}(N, \tilde{\varepsilon})=\bigcup_{p \geq 1} E_{\alpha, p}^{\mu}(N, \tilde{\varepsilon}) .
\end{aligned}
$$


This set contains the points $t$ for which, for every large enough $n$, the $\mu$-measure of the $2 N+1$ neighbors of $I_{n, k_{t}}$ belongs to $\left[b^{-n\left(\beta+\varepsilon_{n}\right)}, b^{-n\left(\beta-\varepsilon_{n}\right)}\right]$. The information on neighboring intervals is involved in the proof of (1.7).

For $n \geq 1$ and $\varepsilon, \eta>0$, consider the quantity

$$
S_{n}^{N, \varepsilon, \eta}(m, \mu, \alpha)=\sum_{\gamma \in\{-1,1\}} b^{n(\alpha-\gamma \varepsilon) \gamma \eta} \sum_{\left\{v, w \in \mathcal{A}^{n}: \delta(v, w) \leq N\right\}} m\left(I_{v}\right) \mu\left(I_{w}\right)^{\gamma \eta} .
$$

The following result was established in [12], but the proof is given here for completeness.

Proposition 2.1. Let $\mu$ and $m$ be two positive Borel measures with supports equal to [0,1]. Let $\tilde{\varepsilon}=\left(\varepsilon_{n}\right)_{n \geq 0}$ be a positive sequence, let $N \geq 1$, and let $\alpha \geq 0$. Let $\left(\eta_{n}\right)_{n \geq 1}$ be a positive sequence. If $\sum_{n \geq 1} S_{n}^{N, \varepsilon_{n}, \eta_{n}}(m, \mu, \alpha)<\infty$, then $E_{\alpha}^{\mu}(N, \tilde{\varepsilon})$ is of full m-measure.

Remark 2.1. Similar conditions were used in [13] to obtain a comparison between the multifractal formalisms of [17] and [46].

Proof of Proposition 2.1. For $\gamma \in\{-1,1\}$ and $n \geq 1$, define

$$
\begin{aligned}
E_{\alpha}^{\mu}\left(N, \varepsilon_{n}, \gamma\right)=\{t \in[0,1]: & b^{\gamma n\left(\alpha-\gamma \varepsilon_{n}\right)} \mu\left(I_{w}\right)^{\gamma} \leq 1 \\
& \text { for all } \left.w \in \mathcal{A}^{n} \text { such that } \delta\left(w, w^{(n)}(t)\right) \leq N\right\} .
\end{aligned}
$$

For $t \in[0,1]$ and $k \in\{-N, \ldots, N\}$, if there exists a (necessarily unique) $w \in \mathcal{A}^{n}$ such that $i(w)-i\left(w^{(n)}(t)\right)=k$, this word $w$ is denoted $w_{k}^{(n)}(t)$.

For $\gamma \in\{-1,1\}$, let $S_{n, \gamma}=\sum_{-N \leq k \leq N} m_{k, n}$ with

$$
m_{k, n}=m\left(\left\{t \in[0,1]: i(w)-i\left(w^{(n)}(t)\right)=k \text { implies that } b^{\gamma n\left(\alpha-\gamma \varepsilon_{n}\right)} \mu\left(I_{w}\right)^{\gamma}>1\right\}\right) .
$$

We clearly have

$$
m\left(E_{\alpha}^{\mu}\left(N, \varepsilon_{n},-1\right)^{\mathrm{c}} \cup E_{\alpha}^{\mu}\left(N, \varepsilon_{n}, 1\right)^{\mathrm{c}}\right) \leq S_{n,-1}+S_{n, 1} .
$$

Fix $\eta_{n}>0$ and $k,-N \leq k \leq N$. Let $Y(t)$ be the random variable defined to equal

$$
b^{\gamma n\left(\alpha-\gamma \varepsilon_{n}\right) \eta_{n}} \mu\left(I_{w_{k}^{(n)}(t)}\right)^{\gamma \eta_{n}}
$$

if $w_{k}^{(n)}(t)$ exists or to equal 0 otherwise. The Markov inequality applied to $Y(t)$ with respect to $m$ yields $m_{k, n} \leq \int Y(t) \mathrm{d} m(t)$. Since $Y$ is constant over each $b$-adic interval $I_{v}$ of generation $n$, we obtain

$$
m_{k, n} \leq \sum_{\left\{v, w \in \mathcal{A}^{n}: i(w)-i(v)=k\right\}} b^{n\left(\alpha-\gamma \varepsilon_{n}\right) \gamma \eta_{n}} m\left(I_{v}\right) \mu\left(I_{w}\right)^{\gamma \eta_{n}} .
$$

Summing over $k \in\{-N, \ldots, N\}$ yields $S_{n,-1}+S_{n, 1} \leq S_{n}^{N, \varepsilon_{n}, \eta_{n}}(m, \mu, \alpha)$. The conclusion follows from (2.3) and from the Borel-Cantelli lemma.

\subsection{Uniform growth speed in singularity sets}

Let $\Lambda$ be a set of indices and $\Omega^{*}$ a measurable subset of $\Omega$ of probability 1 . Some notation and technical assumptions are needed to state the general result that we shall apply in Section 3 . These assumptions describe a common situation in multifractal analysis. In particular, the measures in the following sections satisfy these requirements. 
- For every $\omega \in \Omega^{*}$, we consider two sequences of families of measures, $\left(\left\{\mu_{\lambda}^{w}\right\}_{\lambda \in \Lambda}\right)_{w \in \mathcal{A}^{*}}$ and $\left(\left\{m_{\lambda}^{w}\right\}_{\lambda \in \Lambda}\right)_{w \in \mathcal{A}^{*}}$ (indexed by $\left.\mathcal{A}^{*}\right)$, such that, for every $w \in \mathcal{A}^{*}$, the elements of the families $\left\{\mu_{\lambda}^{w}\right\}_{\lambda \in \Lambda}$ and $\left\{m_{\lambda}^{w}\right\}_{\lambda \in \Lambda}$ are positive, finite Borel measures whose support is $[0,1]$. We write $\left\{\mu_{\lambda}^{\varnothing}\right\}_{\lambda \in \Lambda}$ as $\left\{\mu_{\lambda}\right\}_{\lambda \in \Lambda}$ and we write $\left\{m_{\lambda}^{\varnothing}\right\}_{\lambda \in \Lambda}$ as $\left\{m_{\lambda}\right\}_{\lambda \in \Lambda}$.

- We consider an integer $N \geq 1$, a positive sequence $\tilde{\varepsilon}=\left(\varepsilon_{n}\right)_{n \geq 1}$, and a family of positive numbers $\left\{\alpha_{\lambda}\right\}_{\lambda \in \Lambda}$. Then, recalling (2.2), for every $j \geq 0, w \in \mathcal{A}^{j}$, and $p \geq 1$ we consider the sets

$$
E_{\alpha_{\lambda}, p}^{\mu_{\lambda}^{w}}(N, \tilde{\varepsilon})=\bigcap_{n \geq p} E_{\alpha_{\lambda}}^{\mu_{\lambda}^{w}}\left(N, \varepsilon_{n},-1\right) \cap E_{\alpha_{\lambda}}^{\mu_{\lambda}^{w}}\left(N, \varepsilon_{n}, 1\right) .
$$

The sets $\left\{E_{\alpha_{\lambda}, n}^{\mu_{\lambda}^{w}}(N, \tilde{\varepsilon})\right\}_{n}$ form a nondecreasing sequence. We assume that the measure $m_{\lambda}^{w}$ is concentrated on $\lim _{p \rightarrow \infty} E_{\alpha_{\lambda}, p}^{\mu_{\lambda}^{w}}(N, \tilde{\varepsilon})$. We define the growth speed of $E_{\alpha_{\lambda}, p}^{\mu_{\lambda}^{w}}(N, \tilde{\varepsilon})$ as

$$
G S\left(m_{\lambda}^{w}, \mu_{\lambda}^{w}, \alpha_{\lambda}, N, \tilde{\varepsilon}\right)=\inf \left\{p \geq 1: m_{\lambda}^{w}\left(E_{\alpha_{\lambda}, p}^{\mu_{\lambda}^{w}}(N, \tilde{\varepsilon})\right) \geq\left\|m_{\lambda}^{w}\right\| / 2\right\} .
$$

Since

$$
\mu_{\lambda}^{w}\left(\lim _{p \rightarrow \infty} E_{\alpha_{\lambda}, p}^{\mu_{\lambda}^{w}}(N, \tilde{\varepsilon})\right)=\left\|m_{\lambda}^{w}\right\|
$$

we have

$$
G S\left(m_{\lambda}^{w}, \mu_{\lambda}^{w}, \alpha_{\lambda}, N, \tilde{\varepsilon}\right)<\infty .
$$

This number is a measure of the number, $p$, of generations needed for $E_{\alpha_{\lambda}, p}^{\mu_{\lambda}^{w}}(N, \tilde{\varepsilon})$ to recover a certain given fraction (here chosen to equal $\frac{1}{2}$ ) of the measure $m_{\lambda}^{w}$.

- We assume that, for every positive sequence $\tilde{\eta}=\left(\eta_{j}\right)_{j \geq 0}$, there exist a random vector $(U(\tilde{\eta}), V(\tilde{\eta})) \in \mathbb{R}_{+} \times \mathbb{R}_{+}^{\mathbb{N}}$, a sequence $\left(U^{w}, V^{w}=\left(V_{n}^{w}\right)_{n \in \mathbb{N}}\right)_{w \in A^{*}}$ of copies of $(U(\tilde{\eta}), V(\tilde{\eta}))$, and a sequence $\left(\psi_{j}(\tilde{\eta})\right)_{j \geq 0}$ such that, for P-almost every $\omega \in \Omega^{*}$,

$$
\begin{aligned}
U^{w} \leq \inf _{\lambda \in \Lambda}\left\|m_{\lambda}^{w}\right\|, \quad V_{n}^{w} \geq \sup _{\lambda \in \Lambda} S_{n}^{N, \varepsilon_{n}, \eta_{n}}\left(m_{\lambda}^{w}, \mu_{\lambda}^{w}, \alpha_{\lambda}\right), \\
\quad \text { for all } w \in \mathcal{A}^{*} \text { and all } n \geq \psi_{|w|}(\tilde{\eta}),
\end{aligned}
$$

where the quantity $S_{n}^{N, \varepsilon_{n}, \eta_{n}}\left(m_{\lambda}^{w}, \mu_{\lambda}^{w}, \alpha_{\lambda}\right)$ is as defined in (2.1) (remember that $|w|=j$ if $w \in \mathcal{A}_{j}$ ). This provides us with a uniform control over $\lambda \in \Lambda$ of the families of measures $\left\{\left(m_{\lambda}^{w}, \mu_{\lambda}^{w}\right)\right\}_{w \in \mathcal{A}^{*}}$.

Proposition 2.2. (Uniform growth speed in singularity sets.) Assume that two sequences of positive numbers $\tilde{\eta}=\left(\eta_{j}\right)_{j \geq 0}$ and $\left(\rho_{j}\right)_{j \geq 0}$ are fixed, and let $\left(\delta_{j}\right)_{j \geq 0}$ be a sequence of integers such that $\S_{j} \geq \psi_{j}(\tilde{\eta})$. If

$$
\sum_{j \geq 0} b^{j} \mathrm{P}\left(U(\tilde{\eta}) \leq b^{-\rho_{j}}\right)<\infty \text { and } \sum_{j \geq 0} b^{j} b^{\rho_{j}} \sum_{n \geq \gamma_{j}} \mathrm{E}\left(V_{n}(\tilde{\eta})\right)<\infty,
$$

then, with probability 1 , and for every large enough $j$, every $w \in \mathcal{A}^{j}$, and $\lambda \in \Lambda$,

$$
G S\left(m_{\lambda}^{w}, \mu_{\lambda}^{w}, \alpha_{\lambda}, N, \tilde{\varepsilon}\right) \leq \wp_{j}
$$


Proof. Fix $j \geq 1$ and $w \in \mathcal{A}^{j}$. As shown in the proof of Proposition 2.1, for every $n \geq 1$ and every $\lambda \in \Lambda$ we can write

$$
m_{\lambda}^{w}\left(E_{\alpha_{\lambda}}^{\mu_{\lambda}^{w}}\left(N, \varepsilon_{n},-1\right)^{\mathrm{c}} \cup E_{\alpha_{\lambda}}^{\mu_{\lambda}^{w}}\left(N, \varepsilon_{n}, 1\right)^{\mathrm{c}}\right) \leq S_{n}^{N, \varepsilon_{n}, \eta_{n}}\left(m_{\lambda}^{w}, \mu_{\lambda}^{w}, \alpha_{\lambda}\right) .
$$

Thus, using (2.5), we obtain

$$
m_{\lambda}^{w}\left(\bigcup_{n \geq s_{j}}\left(E_{\alpha_{\lambda}}^{\mu_{\lambda}^{w}}\left(N, \varepsilon_{n},-1\right)^{\mathrm{c}} \cup E_{\alpha_{\lambda}}^{\mu_{\lambda}^{w}}\left(N, \varepsilon_{n}, 1\right)^{\mathrm{c}}\right)\right) \leq \sum_{n \geq s_{j}} V_{n}^{w} .
$$

We apply the 'random self-similar control' (2.6) combined with the Borel-Cantelli lemma. On the one hand, the left-hand relation of (2.6) yields

$$
\sum_{j \geq 1} \mathrm{P}\left(\text { there exists a } w \in \mathcal{A}^{j} \text { such that } U^{w} \leq b^{-\rho_{j}}\right)<\infty .
$$

Hence, with probability 1 , for large enough $j$ and for all $w \in \mathcal{A}^{j}$,

$$
\sup _{\lambda \in \Lambda}\left\|m_{\lambda}^{w}\right\|^{-1} \leq\left(U^{w}\right)^{-1} \leq b^{\rho_{j}}
$$

On the other hand, the right-hand relation of (2.6) yields

$$
\sum_{j \geq 1} \mathrm{P}\left(\text { there exists a } w \in \mathcal{A}^{j} \text { such that } b^{\rho_{j}} \sum_{n \geq \gamma_{j}} V_{n}^{w} \geq \frac{1}{2}\right) \leq 2 \sum_{j \geq 1} b^{j} b^{\rho_{j}} \mathrm{E}\left(\sum_{n \geq s_{j}} V_{n}^{w}\right)<\infty \text {. }
$$

This implies that, with probability $1, b^{\rho_{j}} \sum_{n \geq s_{j}} V_{n}^{w}<\frac{1}{2}$ for every large enough $j$ and all $w \in \mathcal{A}^{j}$.

Thus, by (2.8), $\sup _{\lambda \in \Lambda} \sum_{n \geq s_{j}} V_{n}^{w} /\left\|m_{\lambda}^{w}\right\|<\frac{1}{2}$. By combining this with (2.7) and (2.4), we find that

$$
G S\left(m_{\lambda}^{w}, \mu_{\lambda}^{w}, \alpha_{\lambda}, N, \tilde{\varepsilon}\right) \leq \wp_{j}
$$

for every $\lambda \in \Lambda$.

\section{Main results for independent random cascades}

\subsection{Definition}

Let $v=\left(v_{1}, \ldots, v_{|v|}\right) \in \mathcal{A}^{*}$. For every $k \in\{1, \ldots,|v|\}, v \mid k$ is the truncated word $\left(v_{1}, \ldots, v_{k}\right) \in \mathcal{A}^{k}$, and by convention $v \mid 0$ is the empty word, $\varnothing$. If $v$ and $w$ belong to $\mathcal{A}^{*}$ then the word obtained by concatenation of $v$ and $w$ is denoted by either $v w$ or $v \cdot w$.

In this paper we focus on the measures introduced in [39] and, more recently, in [5]. A measure $\mu(\omega)$ is said to be an independent random cascade if it has the following property. There exist a sequence of random positive vectors $\left(W(w)=\left(W_{0}(w), \ldots, W_{b-1}(w)\right)\right)_{w \in \mathcal{A}^{*}}$ and a sequence of random measures $\left(\mu^{w}\right)_{w \in \mathcal{A}^{*}}$ such that

(P1) for all $v, w \in \mathcal{A}^{*}, \mu\left(I_{v w}\right)=\mu^{v}\left(I_{w}\right) \prod_{k=0}^{|v|-1} W_{v_{k+1}}(v \mid k)$ (with $\left.\mu^{\varnothing}=\mu\right)$;

(P2) the random vectors $W(w), w \in \mathcal{A}^{*}$, are independent and identically distributed with a vector $W=\left(W_{0}, \ldots, W_{b-1}\right)$ such that $\sum_{k=0}^{b-1} \mathrm{E}\left(W_{k}\right)=1$;

(P3) for all $v \in \mathcal{A}^{*},\left(\mu^{v}\left(I_{w}\right)\right)_{w \in \mathcal{A}^{*}} \equiv\left(\mu\left(I_{w}\right)\right)_{w \in \mathcal{A}^{*}}$, and for every $j \geq 1$ the measures $\mu^{v}, v \in \mathcal{A}^{j}$, are mutually independent; and 
(P4) for every $j \geq 1$, the $\sigma$-algebras $\sigma\left(W(w): w \in \bigcup_{0 \leq k \leq j-1} \mathcal{A}^{k}\right)$ and $\sigma\left(\mu^{v}\left(I_{w}\right): v \in \mathcal{A}^{j}\right.$, $\left.w \in \mathcal{A}^{*}\right)$ are independent.

Let $(W(w))_{w \in \mathcal{A}^{*}}$ be as above. For $q \in \mathbb{R}$, define the function

$$
\tilde{\tau}_{\mu}(q)=-\log _{b}\left(\mathrm{E}\left(\sum_{k=0}^{b-1} W_{k}^{q}\right)\right) \in \mathbb{R} \cup\{-\infty\} .
$$

In the following we consider two classes of measures.

- For $\tilde{\tau}_{\mu}^{\prime}\left(1^{-}\right)>0$, we consider nondegenerate multiplicative martingales. Let $(W(w)=$ $\left.\left(W_{0}(w), \ldots, W_{b-1}(w)\right)\right)_{w \in \mathcal{A}^{*}}$ be a sequence of random positive vectors satisfying (P2). With probability 1 , for all $v \in \mathcal{A}^{*}$ the sequence of measures $\left(\mu_{j}^{v}\right)_{j \geq 0}$ defined on $[0,1]$ by

$$
\frac{\mathrm{d} \mu_{j}^{v}}{\mathrm{~d} \ell}(t)=b^{j} \prod_{k=0}^{j-1} W_{t_{k+1}}(v \cdot t \mid k)
$$

converges weakly, as $n \rightarrow \infty$, to a measure $\mu^{v}$. The following statements hold.

1. For $\mu=\mu^{\varnothing}$, properties (P1) to (P4) are satisfied.

2. For $\mu=\mu^{\varnothing}$, the total masses $\left\|\mu^{v}\right\|$ are almost surely positive and their expectations are equal to 1 (see [31] and [20]).

- In the critical case, $\tilde{\tau}_{\mu}^{\prime}\left(1^{-}\right)=0$, we consider the following modified construction. Suppose that $\tilde{\tau}_{\mu}^{\prime}\left(1^{-}\right)=0$ and that $\tilde{\tau}_{\mu}(h)>-\infty$ for some $h>1$. Then, with probability 1 , for all $v \in \mathcal{A}^{*}, \mu^{v}\left(I_{w}\right)=\lim _{j \rightarrow \infty}\left(-H_{j}^{v}(w)\right)$, where

$$
H_{j}^{v}(w)=\sum_{u \in \mathcal{A}^{j}}\left(\prod_{k=0}^{|w|+j-1} W_{(w \cdot u)_{k+1}}(v \cdot(w \cdot u \mid k))\right) \log \left(\prod_{k=0}^{|w|+j-1} W_{(w \cdot u)_{k+1}}(v \cdot(w \cdot u \mid k))\right)
$$

defines a function on the $b$-adic intervals $I_{w}$ which is a positive Borel measure with support [0,1] (see [5] and [37]). The following statements hold.

1. For $\mu=\mu^{\varnothing}$, properties (P1) to (P4) are satisfied.

2. For $\mu=\mu^{\varnothing}, \mathrm{E}\left(\|\mu\|^{h}\right)<\infty$ for $h \in[0,1)$ but $\mathrm{E}(\|\mu\|)=\infty$.

\subsection{Analyzing measures}

Let $\mathcal{O}$ be the interior of the interval $\left\{q \in \mathbb{R}: \tilde{\tau}_{\mu}^{\prime}(q) q-\tilde{\tau}_{\mu}(q)>0\right\}$. We always have $(0,1) \subset \mathcal{O}$, and $\mathcal{O} \subset(-\infty, 1)$ if $\tilde{\tau}_{\mu}^{\prime}\left(1^{-}\right)=0$. We assume that

- if $\tilde{\tau}_{\mu}^{\prime}\left(1^{-}\right)>0$ then $\mathcal{O}$ contains the closed interval $[0,1]$, and that

- if $\tilde{\tau}_{\mu}^{\prime}\left(1^{-}\right)=0$ then $0 \in \mathcal{O}$.

For $q \in \mathcal{O}, v \in \mathcal{A}^{*}$, and $j \geq 1$, let $\mu_{q, j}^{v}$ be the measure defined as $\mu_{j}^{v}$ in (3.2) but with the sequence $\left(W_{q}(v \cdot w)=\left(b^{\tilde{\tau}_{\mu}(q)} W_{0}(v \cdot w)^{q}, \ldots, b^{\tilde{\tau}_{\mu}(q)} W_{b-1}(v \cdot w)^{q}\right)\right)_{w}$ instead of $(W(v \cdot w))_{w}$. It was proved in [5] that, on a set $\Omega^{*} \subset \Omega$ of probability 1 , for all $\omega \in \Omega^{*}$, all $v \in \mathcal{A}^{*}$, and all $q \in \mathcal{O}$, the sequence $\left(\mu_{q, j}^{v}\right)$ converges weakly to a positive measure $\mu_{q}^{v}$. 
It was proved in [15] and [5] that, with probability 1,

- the mappings $\mathcal{O} \ni q \mapsto Y_{q}(v)$ are analytic and positive, where

$$
\mu_{q}^{\varnothing}=\mu_{q}, \quad Y_{q}=\left\|\mu_{q}^{\varnothing}\right\|, \quad \text { and } \quad Y_{q}(v)=\left\|\mu_{q}^{v}\right\| \quad \text { for } v \in \mathcal{A}^{*},
$$

and that

- $\tau_{\mu} \equiv \tilde{\tau}_{\mu}$ on $\mathcal{O}$.

Finally, we remark that $\mathbb{R}_{+} \subset \mathcal{O}$ and $\mathbb{R}_{-} \subset \mathcal{O}$ if and only if, for all $q \in \mathbb{R}_{+}$and, respectively, $q \in \mathbb{R}_{-}, \tilde{\tau}_{\mu}(h q)-h \tilde{\tau}_{\mu}(q)>0$ and $h>1$, which amounts to saying that, for all $q \in \mathbb{R}_{+}$and, respectively, $q \in \mathbb{R}_{-}, \mathrm{E}\left(Y_{q}^{h}\right)<\infty$ and $h>1$ (see the proof of Lemma 4.3).

\subsection{Main results}

For an independent random cascade $\mu$, we assume that if $\tilde{\tau}_{\mu}^{\prime}\left(1^{-}\right)>0$ then $\mathcal{O}$ contains $[0,1]$, and that if $\tilde{\tau}_{\mu}^{\prime}\left(1^{-}\right)=0$ then $\mathcal{O} \subset(-\infty, 1)$. We also suppose that $0 \in \mathcal{O}$.

Theorem 3.1. Let $\mu$ be an independent random cascade. Let $N$ be an integer greater than or equal to 1 , and let $\tilde{\varepsilon}=\left(\varepsilon_{n}\right)_{n \geq 1}$ be a sequence of positive numbers decreasing to 0 . Assume that the series $\sum_{n \geq 1} n b^{-n \chi \varepsilon_{n}^{2}}$ converges, for all $\chi>0$.

Then, with probability 1 for every $q \in \mathcal{O}, \tau_{\mu}(q)=\tilde{\tau}_{\mu}(q)$ and the level sets $E_{\tau_{\mu}^{\prime}(q)}^{\mu}(N, \tilde{\varepsilon})$ and $E_{\tau_{\mu}^{\prime}(q) q-\tau_{\mu}(q)}^{\mu_{q}}(N, \tilde{\varepsilon})$ are both of full $\mu_{q}$-measure.

Remark 3.1. The conclusions of Theorem 3.1 hold if

$$
\text { there exists an } \eta>0 \text { such that } \varepsilon_{n} \geq n^{-1 / 2} \log (n)^{1 / 2+\eta} \text { for every } n \text {. }
$$

This condition on $\left(\varepsilon_{n}\right)_{n \geq 1}$ must be compared with the stronger one which holds if we consider only one measure, $\mu_{q}$, generated by a vector $W_{q}=W_{q}(\varnothing)$ which satisfies the additional conservative condition $\sum_{k=0}^{b-1} W_{q, k}=1$. Indeed, in this case, $Y_{q}=1$ almost surely and, as a function of $(t, \omega)$, the logarithmic density $\log \left(\mu_{q}\left(I_{n}(t)\right)\right) / n$ is a sum of independent, identically distributed random variables with respect to the probability measure $\mathcal{Q}_{q}$, to be introduced in Section 4.4. If $0<\sigma_{q}^{2}=\sum_{k=0}^{b-1} W_{q, k} \log ^{2} W_{q, k}<\infty$, then the law of the iterated logarithm implies that, with probability 1 ,

$$
\limsup _{n \rightarrow \infty}\left|\frac{\log \mu_{q}\left(I_{n}(t)\right)-n\left(\tau_{\mu}^{\prime}(q) q-\tau_{\mu}(q)\right)}{\sigma_{q} \sqrt{n \log \log (n)}}\right|=1 \quad \mu_{q} \text {-almost everywhere. }
$$

In this case, for $n \geq 3, \varepsilon_{n}$ can be chosen to equal $\sigma^{\prime} \sqrt{n \log \log (n)}$ for any $\sigma^{\prime}>\sigma_{q}$.

The estimate (3.4) comes from our wish to control the asymptotic behavior of an uncountable family of measures simultaneously, and from the technique we use. Moreover, when $W_{q}$ does not satisfy the conservative condition described above, it seems difficult to control the asymptotic behavior using a term of the form $\sigma^{\prime} \sqrt{|v| \log \log (|v|)}$.

Theorem 3.2. (Growth speed in Hölder singularity sets.) Under the assumptions of Theorem 3.1, assume that $\left(\varepsilon_{n}\right)_{n}$ satisfies (3.4) and that there exists an $A>1$ such that, with probability $1, A^{-1} \leq W_{i}$ or $W_{i} \leq A$ for all $i, 0 \leq i \leq b-1$, and let $K$ be a compact subinterval of $\mathcal{O} \cap \mathbb{R}_{+}$or, respectively, $\mathcal{O} \cap \mathbb{R}_{-}$. Then, with probability 1 , for large enough $j$, all $q \in K$, and $w \in \mathcal{A}^{j}$,

$$
\max \left(G S\left(\mu_{q}^{w}, \mu^{w}, \tau_{\mu}^{\prime}(q), N, \tilde{\varepsilon}\right), G S\left(\mu_{q}^{w}, \mu_{q}^{w}, \tau_{\mu}^{\prime}(q) q-\tau_{\mu}(q), N, \tilde{\varepsilon}\right)\right) \leq s_{j},
$$

with $\varsigma_{j}=\left[\exp \left(\left(j \log (j)^{\eta}\right)^{1 /(1+2 \eta)}\right)\right]$ (where $[\cdot]$ denotes the integer-part function). 
If there exists an $\eta>0$ such that, for every $n, \varepsilon_{n} \geq \log (n)^{-\eta}$, then the above conclusion holds with $\S_{j}=\left[j \log (j)^{\eta^{\prime}}\right]$, for any $\eta^{\prime}>2 \eta$.

Remark 3.2. Our computations show that, the faster $\left(\varepsilon_{n}\right)$ decreases with $n$, the faster the growth speed increases (in the sense that $\delta_{j}$ increases faster than $j$ ). In Theorem 3.2, the first choice of $\left(\varepsilon_{n}\right)$ corresponds to the fastest choice for the convergence speed of $\left(\varepsilon_{n}\right)$ allowed by our technique (see Remark 3.1). As a counterpart, $s_{j}$ is very large compared to $j$. The second choice for $\left(\varepsilon_{n}\right)$ is the slowest one, but as a counterpart $\delta_{j}$ corresponds to the 'best' choice allowed to minimize $s_{j} / j$.

Using the proof of Theorem 3.2, we can find other pairs $\left(\left(\delta_{j}\right)_{j \geq 1},\left(\varepsilon_{n}\right)_{n \geq 1}\right)$ for which (3.5) holds and whose asymptotic behaviors are intermediate between those of the 'extremal' pairs presented in the statement.

Remark 3.3. We assume that the number of neighbors, $N$, is fixed. In fact, it is not difficult to consider a sequence of $N_{n}$ neighbors simultaneously with the speed of convergence $\varepsilon_{n}$. The number $N_{n}$ can then go to $\infty$ under the condition that $\log N_{n}=o\left(n \varepsilon_{n}^{2}\right)$. Another modification would consist in replacing the fixed fraction $f$ in (1.10) by a fraction $f_{j}$ going to 1 as $j$ goes to $\infty$. The choice $f_{j}=1-b^{-s_{j}}$ with $s_{j}=j$ is convenient. These two improvements yield technical complications, but comparable results are easily derived from the proofs we propose.

The growth speed obtained in Theorem 3.2 can be improved by considering results valid only almost surely, for almost every $q, \mu_{q}$-almost everywhere. Recall that if $t \in[0,1)$ and $j \geq 1$, then $w^{(j)}(t)$ is the unique $w \in \mathcal{A}^{j}$ such that $t \in\left[i(w) b^{-j},(i(w)+1) b^{-j}\right)$.

Theorem 3.3. (Improved growth speed.) Under the assumptions of Theorem 3.1, fix a $\kappa>0$ and assume that (3.4) holds. For $j \geq 2$, let $\S_{j}=\left[j \log (j)^{-\kappa}\right]$.

1. For every $q \in \mathcal{O}$, with probability 1 the following property (property $\mathcal{P}(q)$ ) holds. For $\mu_{q}$-almost every $t \in[0,1)$, if $j$ is large enough then, for $w=w^{(j)}(t)$,

$$
\max \left(G S\left(\mu_{q}^{w}, \mu^{w}, \tau_{\mu}^{\prime}(q), N, \tilde{\varepsilon}\right), G S\left(\mu_{q}^{w}, \mu_{q}^{w}, \tau_{\mu}^{\prime}(q) q-\tau_{\mu}(q), N, \tilde{\varepsilon}\right)\right) \leq s_{j} .
$$

2. With probability 1 , property $\mathcal{P}(q)$ holds for almost every $q \in \mathcal{O}$.

For $w \in \mathcal{A}^{*}, n \geq 1$, and $q \in \mathcal{O}$, let

$$
\mathcal{N}_{n}\left(\mu^{w}, \alpha, \varepsilon_{n}\right)=\operatorname{card}\left\{b \text {-adic box } I \text { of scale } n:|I|^{\alpha+\varepsilon_{n}} \leq \mu^{w}(I) \leq|I|^{\alpha-\varepsilon_{n}}\right\},
$$

where $|I|$ denotes the diameter of $I$. Remember that $\tau_{\mu}=\tilde{\tau}_{\mu}$ on $\mathcal{O}$.

Theorem 3.4. (Renewal speed of the large deviation spectrum.) Under the assumptions of Theorem 3.1, we also assume that (3.4) holds, that $\mathcal{O}=\mathbb{R}$ (in particular, $\left.\tilde{\tau}_{\mu}^{\prime}(1)>0\right)$, and that there exists an $A>1$ such that, with probability $1, A^{-1} \leq W_{i} \leq A$ for all $i, 0 \leq i \leq$ $b-1$. Let $s_{j}$ be as defined in Theorem 3.2, let $K$ be a compact subinterval of $\mathbb{R}$, and let $\beta=1+\max _{q \in K}|q|$. Then, with probability 1 , for large enough $j$, all $q \in K$, and $w \in \mathcal{A}^{j}$, we have

$$
\begin{array}{r}
Y_{q}(w) b^{n\left(\tilde{\tau}_{\mu}^{\prime}(q) q-\tilde{\tau}_{\mu}(q)-\beta \varepsilon_{n}\right)} \leq \mathcal{N}_{n}\left(\mu^{w}, \tilde{\tau}_{\mu}^{\prime}(q), \varepsilon_{n}\right) \leq Y_{q}(w) b^{n\left(\tilde{\tau}_{\mu}^{\prime}(q) q-\tilde{\tau}_{\mu}(q)+\beta \varepsilon_{n}\right)} \\
\qquad \text { for all } n \geq \delta_{j} .
\end{array}
$$


Heuristically, Theorem 3.4 asserts that, for large values of $j$, if $|w|=j$ then the number of $b$-adic intervals of scale $b^{-n}$ such that $\mu^{w}(I) \sim|I|^{\tau_{\mu}^{\prime}(q)}$ approximately equals $b^{n\left(\tilde{\tau}_{\mu}^{\prime}(q) q-\tilde{\tau}_{\mu}(q)\right)}$ for $n \geq \diamond_{j}$. Hence, (3.6) contains precise information on the renewal of the large deviation spectrum $\alpha \mapsto \lim _{\varepsilon \rightarrow 0} \lim _{j \rightarrow \infty} j^{-1} \log _{b} N_{j}^{\varepsilon}(\alpha)$ (see (1.2)).

For $w \in \mathcal{A}^{*}, n \geq 1$, and $q \in \mathbb{R}$, introduce the functions

$$
\tau_{n}^{w}(q)=-\frac{1}{n} \log _{b}\left(\sum_{v \in \mathfrak{A}^{n}} \mu^{w}\left(I_{v}\right)^{q}\right)
$$

( $\tau_{n}^{\varnothing}$ is associated with $\mu^{\varnothing}=\mu$ and is simply denoted by $\tau_{n}$ ).

Theorem 3.5. (Convergence speed of $\tau_{n}^{w}$ toward $\tilde{\tau}_{\mu}$.) Under the assumptions of Theorem 3.4, let $K$ be a compact subinterval of $\mathbb{R}$. There exist $\theta>0$ and $\delta \in(0,1)$ such that, with probability 1 ,

1. for large enough $j,\left|\tilde{\tau}_{\mu}(q)-\tau_{j}(q)\right| \leq\left|\log _{b} Y_{q}\right| j^{-1}+\theta \log (j) j^{-1}$, and

2. for large enough $j$, every $n \geq j^{\delta}$, and every $w \in \mathcal{A}^{j}$,

$$
\left|\tilde{\tau}_{\mu}(q)-\tau_{n}^{w}(q)\right| \leq\left|\log _{b} Y_{q}(w)\right| n^{-1}+\theta \log (n) n^{-1},
$$

with $\left|\log Y_{q}(w)\right| \leq \theta \log j$.

The convergence speed obtained in Theorem 3.5 provides precise information on the estimator of the function $\tau_{\mu}$ discussed in [18] and [47].

\section{Proofs of the main results}

\subsection{Proof of Theorem 3.1}

Fix $K$, a compact subinterval of $\mathcal{O}$, and $\tilde{\eta}=\left(\eta_{n}\right)_{n \geq 1}$, a bounded, positive sequence to be specified later. For $\omega \in \Omega^{*}$ and $q \in K$, introduce

$$
F_{n}(q)=S_{n}^{N, \varepsilon_{n}, \eta_{n}}\left(\mu_{q}, \mu, \tau_{\mu}^{\prime}(q)\right) \quad \text { and } \quad G_{n}(q)=S_{n}^{N, \varepsilon_{n}, \eta_{n}}\left(\mu_{q}, \mu_{q}, \tau_{\mu}^{\prime}(q)-\tau_{\mu}(q)\right)
$$

(recall (2.1)). We begin by giving estimates for $\mathrm{E}\left(H_{n}(q)\right)$ and $\mathrm{E}\left(H_{n}^{\prime}(q)\right)$, for $H \in\{F, G\}$.

Lemma 4.1. Under the assumptions of Theorem 3.1, for small enough $\|\tilde{\eta}\|_{\infty}$ there exists a $C_{K}>0$ such that

$$
\max \left(\mathrm{E}\left(H_{n}(q)\right), \mathrm{E}\left(H_{n}^{\prime}(q)\right)\right) \leq C_{K} n b^{-n\left(\varepsilon_{n} \eta_{n}+O\left(\eta_{n}^{2}\right)\right)} \quad \text { for all } q \in K
$$

with $H \in\{F, G\}$, where $O\left(\eta_{n}^{2}\right)$ is uniform over $q \in K$.

The proof of this lemma is postponed to the next subsection. have

Let $q_{0}$ be the left-hand endpoint of $K$. Since $\sup _{q \in K} H_{n}(q) \leq H_{n}\left(q_{0}\right)+\int_{K}\left|H_{n}^{\prime}(q)\right| \mathrm{d} q$, we

$$
\mathrm{E}\left(\sup _{q \in K} H_{n}(q)\right) \leq C_{K}(1+|K|) n b^{-n\left(\varepsilon_{n} \eta_{n}+O\left(\eta_{n}^{2}\right)\right)} .
$$

Choosing $\eta_{n}=\varepsilon_{n} / A$ for large enough $A$ yields $\varepsilon_{n} \eta_{n}+O\left(\eta_{n}^{2}\right) \geq \varepsilon_{n}^{2} / 2 A$. Under the assumptions of Theorem 3.1, we obtain the almost-sure convergence of $\sum_{n \geq 1} \sup _{q \in K} H_{n}(q)$ for $H \in\{F, G\}$. We then conclude the proof by using Proposition 2.1 . 


\subsection{Proof of Lemma 4.1}

4.2.1. The case $H=F$. For $v, w \in \mathcal{A}^{n}, q \in \mathcal{O}$, and $\gamma \in\{-1,1\}$, we write

$$
\mu_{q}\left(I_{v}\right) \mu\left(I_{w}\right)^{\gamma \eta_{n}}=Y_{q}(v) Y_{1}(w)^{\gamma \eta_{n}} b^{n \tau_{\mu}(q)} \prod_{k=0}^{n-1} W_{v_{k+1}}(v \mid k)^{q} W_{w_{k+1}}(w \mid k)^{\gamma \eta_{n}}
$$

(recall that $Y_{q}(v)$ was defined in (3.3)). Moreover, it follows from estimates of Lemma 6 of [5] that, for small enough $\|\tilde{\eta}\|_{\infty}$, the quantities

$$
\begin{aligned}
& C_{K}^{\prime}(\tilde{\eta})=\sup _{\substack{q \in K, \gamma \in\{-1,1\} \\
n \geq 1, v, w \in \mathcal{A}^{n}}}\left(\mathrm{E}\left(\left|\frac{\mathrm{d}}{\mathrm{d} q} Y_{q}(v) Y_{1}(w)^{\gamma \eta_{n}}\right|\right)+\mathrm{E}\left(Y_{q}(v) Y_{1}(w)^{\gamma \eta_{n}}\right)\right), \\
& C_{K}^{\prime \prime}(\tilde{\eta})=\sup _{\substack{q \in K, \gamma \in\{-1,1\} \\
n \geq 1, v, w \in \mathcal{A}^{n}, 0 \leq k \leq n-1}} \frac{\mathrm{E}\left(\left|(\mathrm{d} / \mathrm{d} q) W_{v_{k+1}}(v \mid k)^{q} W_{w_{k+1}}(w \mid k)^{\gamma \eta_{n}}\right|\right)}{\mathrm{E}\left(W_{v_{k+1}}(v)^{q} W_{w_{k+1}}(w)^{\gamma \eta_{n}}\right)}
\end{aligned}
$$

are finite. Hence, due to the definition of $F_{n}(q)$ and the fact that $\tilde{\tau}$ is continuously differentiable on $\mathcal{O}$, there exists a constant $C_{K}(\tilde{\eta})$ such that, for every $q \in K, \max \left(\mathrm{E}\left(F_{n}(q)\right), \mathrm{E}\left(F_{n}^{\prime}(q)\right)\right) \leq$ $C_{K}(\tilde{\eta}) T_{n}(q)$, where

$$
T_{n}(q)=n b_{n}(q) \sum_{\substack{\gamma \in\{-1,1\} \\ v, w \in \mathcal{A}^{n}, \delta(v, w) \leq N}} \prod_{k=0}^{n-1} \mathrm{E}\left(W_{v_{k+1}}(v \mid k)^{q} W_{w_{k+1}}(w \mid k)^{\gamma \eta_{n}}\right)
$$

with $b_{n}(q)=b^{n\left(\tau_{\mu}(q)+\gamma \eta_{n}\left(\tau_{\mu}^{\prime}(q)-\gamma \varepsilon_{n}\right)\right)}$.

Let us make the following important remark.

Remark 4.1. If $v$ and $w$ are words of length $n$, and if $\bar{v}$ and $\bar{w}$ stand for their prefixes of length $n-1$, then $\delta(\bar{v}, \bar{w})>k$ implies that $\delta(v, w)>b k$. Thus, given two integers, $n$ and $m$, $n \geq m>0$, and two words, $v$ and $w$, in $\mathcal{A}^{n}$ such that $b^{m-1}<\delta(v, w) \leq b^{m}$, there are two prefixes, $\bar{v}$ and $\bar{w}$ (of, respectively, $v$ and $w$ ), of common length $n-m$ such that $\delta(\bar{v}, \bar{w}) \leq 1$. Moreover, there are at most $b^{2 m}$ pairs $(v, w)$ of words in $\mathcal{A}^{n}$ such that $\bar{v}$ and $\bar{w}$ are respectively the prefixes of $v$ and $w$.

According to Remark 4.1 and the form of $T_{n}(q)$, there exists a constant $C_{K}^{\prime \prime \prime}$ such that

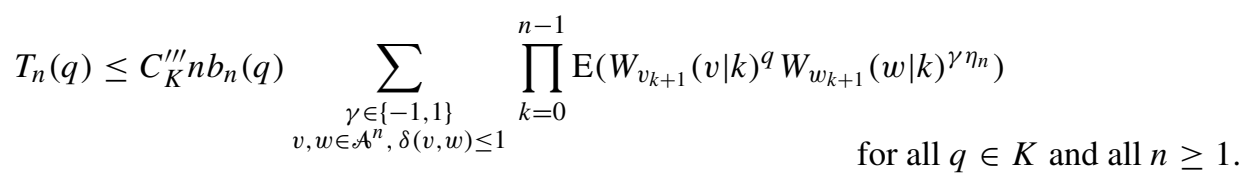

The situation is thus reducible to the case $N=1$. Now, $T_{n}(q) \leq n\left(T_{n, 1}(q)+T_{n, 2}(q)\right)$, where

$$
\begin{aligned}
& T_{n, 1}(q)=b_{n}(q) \sum_{\gamma \in\{-1,1\}, v \in \mathcal{A}^{n}} \prod_{k=0}^{n-1} \mathrm{E}\left(W_{v_{k+1}}(v \mid k)^{q+\gamma \eta_{n}}\right), \\
& T_{n, 2}(q)=b_{n}(q) \sum_{\substack{\gamma \in\{-1,1\} \\
v, w \in \mathcal{A}^{n}, \delta(v, w)=1}} \prod_{k=0}^{n-1} \mathrm{E}\left(W_{v_{k+1}}(v \mid k)^{q} W_{w_{k+1}}(w \mid k)^{\gamma \eta_{n}}\right) .
\end{aligned}
$$


By using the twice continuous differentiability of $\tau_{\mu}\left(\tau_{\mu}=\tilde{\tau}_{\mu}\right)$, we immediately find that

$$
T_{n, 1}(q)=\sum_{\gamma \in\{-1,1\}} b^{n\left(\tau_{\mu}(q)+\gamma \eta_{n}\left(\tau_{\mu}^{\prime}(q)-\gamma \varepsilon_{n}\right)-\tau_{\mu}\left(q+\gamma \eta_{n}\right)\right)}=2 b^{-n\left(\varepsilon_{n} \eta_{n}+O\left(\eta_{n}^{2}\right)\right)},
$$

where $O\left(\eta_{n}^{2}\right)$ is uniform over $q \in K$ if $\|\tilde{\eta}\|_{\infty}$ is small enough.

Let $g_{k}$ and $d_{k}$ respectively denote the word consisting of $k$ consecutive 0 s and the word consisting of $k$ consecutive entries of $b-1$. The estimation of $T_{n, 2}(q)$ is achieved using the following identity:

$$
\bigcup_{v, w \in \mathcal{A}^{n}, \delta(v, w)=1}(v, w)=\bigcup_{m=0}^{n-1} \bigcup_{u \in \mathcal{A}^{m}} \bigcup_{r \in\{0, \ldots, b-2\}}\left(u \cdot r \cdot d_{n-1-m}, u \cdot(r+1) \cdot g_{n-1-m}\right) .
$$

We have $T_{n, 2}(q)=\mathcal{T}_{n}(q,-1)+\mathcal{T}_{n}(q, 1)$, where, for $\gamma \in\{-1,1\}$,

$$
\begin{aligned}
\mathcal{T}_{n}(q, \gamma) & =b_{n}(q) \sum_{\substack{v, w \in \mathcal{A}^{n} \\
\delta(v, w)=1}} \prod_{k=0}^{n-1} \mathrm{E}\left(W_{v_{k+1}}(v \mid k)^{q} W_{w_{k+1}}(w \mid k)^{\gamma \eta_{n}}\right) \\
& =b_{n}(q) \sum_{m=0}^{n-1} \sum_{u \in \mathcal{A}^{m}} \sum_{r=0}^{b-2} \Theta_{n-1-m}(r) \prod_{k=0}^{m-1} \mathrm{E}\left(W_{u_{k+1}}(u \mid k)^{q+\gamma \eta_{n}}\right) \\
& =b_{n}(q) \sum_{m=0}^{n-1} b^{-m \tau_{\mu}\left(q+\gamma \eta_{n}\right)} \sum_{r=0}^{b-2} \Theta_{n-1-m}(r)
\end{aligned}
$$

with

$$
\Theta_{m}(r)=\mathrm{E}\left(W_{r}^{q} W_{r+1}^{\gamma \eta_{n}}\right) \mathrm{E}\left(W_{b-1}^{q}\right)^{m} \mathrm{E}\left(W_{0}^{\gamma \eta_{n}}\right)^{m}+\mathrm{E}\left(W_{r}^{\gamma \eta_{n}} W_{r+1}^{q}\right) \mathrm{E}\left(W_{0}^{q}\right)^{m} \mathrm{E}\left(W_{b-1}^{\gamma \eta_{n}}\right)^{m} .
$$

All the components of $W$ are positive almost surely. Thus, by the definition, (3.1), of $\tilde{\tau}_{\mu}(q)=$ $\tau_{\mu}(q)$, there is a constant $c_{K} \in(0,1)$ such that $\max \left(\mathrm{E}\left(W_{0}^{q}\right), \mathrm{E}\left(W_{b-1}^{q}\right)\right) \leq c_{K} b^{-\tau_{\mu}(q)}$ for all $q \in K$. Moreover, if $\|\tilde{\eta}\|_{\infty}$ is small enough then $\max \left(\mathrm{E}\left(W_{0}^{\gamma \eta_{n}}\right), \mathrm{E}\left(W_{b-1}^{\gamma \bar{\eta}_{n}}\right)\right) \leq\left(c_{K}^{-1}+1\right) / 2$ (this maximum goes to 1 as $\|\eta\|_{\infty}$ goes to 0 ). This yields

$$
\begin{aligned}
\Theta_{m}(r) & \leq\left(\mathrm{E}\left(W_{r}^{q} W_{r+1}^{\gamma \eta_{n}}\right)+\mathrm{E}\left(W_{r}^{\gamma \eta_{n}} W_{r+1}^{q}\right)\right)\left(\frac{c_{K}+1}{2}\right)^{m} b^{-m \tau_{\mu}(q)} \\
& \leq C_{K}\left(\frac{c_{K}+1}{2}\right)^{m} b^{-m \tau_{\mu}(q)}
\end{aligned}
$$

(since $\left.c_{K}\left(c_{K}^{-1}+1\right)=c_{K}+1\right)$. Consequently, we obtain

$$
\begin{aligned}
\mathcal{T}_{n}(q, \gamma) & \leq C_{K} b^{n\left(\tau_{\mu}(q)+\gamma \eta_{n}\left(\tau_{\mu}^{\prime}(q)-\gamma \varepsilon_{n}\right)\right)} b^{-(n-1) \tau_{\mu}\left(q+\gamma \eta_{n}\right)} \sum_{m=0}^{n-1}\left(\frac{c_{K}+1}{2}\right)^{m} b^{m\left(\tau_{\mu}\left(q+\gamma \eta_{n}\right)-\tau_{\mu}(q)\right)} \\
& \leq C_{K} b^{-n\left(\varepsilon_{n} \eta_{n}+O\left(\eta_{n}^{2}\right)\right)} \sum_{m=0}^{n-1}\left(\frac{c_{K}+1}{2}\right)^{m} b^{m\left(\tau_{\mu}\left(q+\gamma \eta_{n}\right)-\tau_{\mu}(q)\right)}
\end{aligned}
$$


The function $\tau_{\mu}$ is continuously differentiable. Hence, the sum

$$
\sum_{m=0}^{n-1}\left(\frac{c_{K}+1}{2}\right)^{m} b^{m\left(\tau_{\mu}\left(q+\gamma \eta_{n}\right)-\tau_{\mu}(q)\right)}
$$

is uniformly bounded over $n \geq 0$ and $q \in K$ if $\|\tilde{\eta}\|_{\infty}$ is small enough. Finally, if $\|\tilde{\eta}\|_{\infty}$ is small enough then we also have $\mathcal{T}_{n}(q, \gamma) \leq C_{K} b^{-n\left(\varepsilon_{n} \eta_{n}+O\left(\eta_{n}^{2}\right)\right)}$. Going back to $T_{n}(q)$, we find that $T_{n}(q) \leq C_{K} n b^{-n\left(\varepsilon_{n} \eta_{n}+O\left(\eta_{n}^{2}\right)\right)}$ for all $q \in K$. This proves (4.1).

4.2.2. The case $H=G$. The proof follows similar lines to that in the previous case. The only additional property required in the computations is the boundedness of $\sup _{q \in K} \mathrm{E}\left(Y_{q}^{-h}\right)$ for some $h>0$. In fact, we shall need the following stronger property in the proof of Theorem 3.2.

Lemma 4.2. 1. If $K \subset \mathcal{O}$ is a compact interval then there exists an $h>0$ such that $\mathrm{E}\left(\sup _{q \in K} Y_{q}^{-h}\right)<\infty$.

2. Assume that there exists an $A>1$ such that, with probability 1 , either $A^{-1} \leq W_{i}$ or $W_{i} \leq A$ for all $i, 0 \leq i \leq b-1$. Then, for any compact interval $K \subset \mathcal{O} \cap \mathbb{R}_{+}$or, respectively, $K \subset \mathcal{O} \cap \mathbb{R}_{-}$, there are two constants, $C_{K}>0$ and $\gamma_{K} \in(0,1)$ (depending on $K$ ), such that

$$
\mathrm{P}\left(\inf _{q \in K} Y_{q} \leq x\right) \leq \exp \left(-C_{K} x^{-\gamma_{K} /\left(1-\gamma_{K}\right)}\right) \quad \text { for all small enough } x>0
$$

Proof. 1. Fix $K$, a compact subinterval of $\mathcal{O} \cap \mathbb{R}_{+}$or $\mathcal{O} \cap \mathbb{R}_{-}$. For $w \in \mathcal{A}^{*}$, we define $Z_{K}(w)=\inf _{q \in K} Y_{q}(w)\left(Z_{K}(\varnothing)\right.$ is denoted by $\left.Z_{K}\right)$. We learn from [15] and [5] that this infimum is positive since $q \mapsto Y_{q}(w)$ is almost surely positive and continuous.

For the random vector $W_{q}(w)=\left(W_{q, 0}(w), W_{q, 1}(w), \ldots, W_{q, b-1}(w)\right)$, define $W_{K}(w)=$ $\inf _{q \in K, 0 \leq i \leq b-1} W_{q, i}(w)\left(W_{K}(\varnothing)\right.$ is denoted by $\left.W_{K}\right)$. Since we assumed that $\mathcal{O}$ contains a neighborhood of 0 , there exists an $h>0$ such that the moment of order $-h$ of this random variable $W_{K}(w)$ is finite.

Moreover, with probability 1 , for all $q \in \mathcal{O}$ we have $Y_{q}=\sum_{i=0}^{b-1} W_{q, i}(\varnothing) Y_{q}(i)$. Hence,

$$
Z_{K} \geq W_{K} \sum_{i=0}^{b-1} Z_{K}(i)
$$

By construction, the random variables $Z_{K}(i), 0 \leq i \leq b-1$, are independent and identically distributed with $Z_{K}$, and are independent of the positive random variable $W_{K}$. Consequently, the Laplace transform of $Z_{K}$, denoted $L: t \mapsto \mathrm{E}\left(\exp \left(-t Z_{K}\right)\right)$ for $t \geq 0$, satisfies the inequality

$$
L(t) \leq \mathrm{E}\left(\prod_{i=0}^{b-1} L\left(W_{K} t\right)\right), \quad \text { for } t \geq 0 .
$$

Since $\mathrm{E}\left(W_{K}^{-h}\right)<\infty$, by using the approach of [43] to study the behavior at $\infty$ of Laplace transforms satisfying an inequality like (4.3) (see also [4] and [38]), we obtain $\mathrm{E}\left(Z_{K}^{-h}\right)<\infty$.

2. The second statement of the lemma is a simple consequence of Theorem 2.5 of [38] (or Corollary 2.5 of [27]) and of the fact that the random variable $W_{K}$ in (4.3) is bounded from below by a positive constant. 


\subsection{Proof of Theorem 3.2}

Fix $K$, a compact subinterval of $\mathcal{O}$. The computations performed to prove Theorem 3.1 yield (4.2). Thus, there are two constants, $C>0$ and $\beta>0$, and a sequence $\tilde{\eta}=\left(\eta_{n}\right)_{n \geq 1} \in \mathbb{R}_{+}^{\mathbb{N}^{*}}$ such that, for every $j, n \geq 1, q \in K$, and $w \in \mathcal{A}^{j}$,

$$
\mathrm{E}\left(\sup _{q \in K} S^{N, \varepsilon_{n}, \eta_{n}}\left(\mu_{q}^{w}, \mu^{w}, \tau_{\mu}^{\prime}(q)\right)\right) \leq C n b^{-\beta n \varepsilon_{n}^{2}} .
$$

In order to apply Proposition 2.2, we take the following steps.

- Let $\Lambda=K,\left\{\left(m_{\lambda}^{w}, \mu_{\lambda}^{w}\right)\right\}_{w \in \mathcal{A}^{*}}=\left\{\left(\mu_{q}^{w}, \mu^{w}\right)\right\}_{w \in \mathcal{A}^{*}, q \in K}$, and $\left\{\alpha_{\lambda}\right\}_{\lambda \in \Lambda}=\left\{\tau_{\mu}^{\prime}(q)\right\}_{q \in K}$.

- For $w \in \mathcal{A}^{*}$ and $n \geq 1$, let

$$
U^{w}=\inf _{q \in K}\left\|\mu_{q}^{w}\right\| \quad \text { and } \quad V_{n}^{w}=\sup _{q \in K} S^{N, \varepsilon_{n}, \eta_{n}}\left(\mu_{q}^{w}, \mu^{w}, \tau_{\mu}^{\prime}(q)\right) .
$$

- For every $j \geq 1$, let $\psi_{j}(\tilde{\eta})=1$ and $\rho_{j}=\log (j)^{1+\eta}$.

- Fix $\eta>0$ and $\eta^{\prime}>2 \eta$. For every $j \geq 1$, let $\&_{j}=\left[\exp \left(\left(j \log (j)^{\eta}\right)^{1 /(1+2 \eta)}\right)\right]$ if $\log (j)^{-\eta} \geq \varepsilon_{j} \geq j^{-1 / 2} \log (j)^{1 / 2+\eta}$ and let $\delta_{j}=\left[j \log (j)^{\eta^{\prime}}\right]$ if $\varepsilon_{j} \geq \log (j)^{-\eta}$.

Now, on the one hand, Lemma 4.2 implies that

$$
u_{j}:=b^{j} \mathrm{P}\left(U^{w} \leq b^{-\rho_{j}}\right) \leq b^{j} \exp \left(-C_{K} b^{\gamma_{K}(\log j)^{(1+\eta)} /\left(1-\gamma_{K}\right)}\right) .
$$

Moreover, $\sum_{j \geq 1} u_{j}<\infty$. On the other hand, for some $\chi>0$ and any $w \in \mathcal{A}^{*}$, we have

$$
v_{j}:=\sum_{n \geq s_{j}} \mathrm{E}\left(V_{n}^{w}\right) \leq \sum_{n \geq s_{j}} C n b^{-\beta n \varepsilon_{n}^{2}}=O\left(b^{-j \log (j)^{\chi}}\right) .
$$

The sequence $\rho_{j}$ has been chosen so that $\sum_{j \geq 1} b^{j} b^{\rho_{j}} v_{j}<\infty$. Consequently, Proposition 2.2 yields the desired upper bound for the growth speed $G S\left(\mu_{q}^{w}, \mu^{w}, \tau_{\mu}^{\prime}(q), N, \tilde{\varepsilon}\right)$.

Upon replacing the measures $\left\{\left(m_{\lambda}^{w}, \mu_{\lambda}^{w}\right)\right\}_{w \in \mathcal{A}^{*}}$ by $\left\{\mu_{q}^{w}, \mu_{q}^{w}\right\}_{w \in \mathcal{A}^{*}, q \in K}$ and replacing the exponents $\left\{\tau_{\mu}^{\prime}(q)\right\}_{q \in K}$ by $\left\{\tau_{\mu}^{\prime}(q) q-\tau_{\mu}(q)\right\}_{q \in K}$, the same arguments yield the conclusion for $G S\left(\mu_{q}^{w}, \mu_{q}^{w}, \tau_{\mu}^{\prime}(q) q-\tau_{\mu}(q), N, \tilde{\varepsilon}\right)$.

\subsection{Proof of Theorem 3.3}

We prove only the results for the control of $G S\left(\mu_{q}^{w}, \mu^{w}, \tau_{\mu}^{\prime}(q), N, \tilde{\varepsilon}\right)$ by $\S_{j}$, since

$$
\left.G S\left(\mu_{q}^{w}, \mu_{q}^{w}, \tau_{\mu}^{\prime}(q) q-\tau_{\mu}(q)\right), N, \tilde{\varepsilon}\right)
$$

is controlled using the same approach.

We first prove part 1 of the theorem. Recall that $(\Omega, \mathcal{B}, \mathrm{P})$ denotes the probability space on which the random variables are defined. On $\mathscr{B} \otimes \mathscr{B}([0,1])$ we consider the so-called Peyrière probability $\mathcal{Q}_{q}[31]$, such that

$$
\mathcal{Q}_{q}(A)=\mathrm{E}\left(\int_{[0,1]} \mathbf{1}_{A}(\omega, t) \mu_{q}(\mathrm{~d} t)\right), \quad A \in \mathscr{B} \otimes \mathscr{B}([0,1]) .
$$

By construction, ' $Q_{q}$-almost surely' means 'P-almost surely, $\mu_{q}(\omega)$-almost everywhere'. 
Fix $\tilde{\eta}$ as in the proof of Theorem 3.2. Also, for $j \geq 1$ let $\rho_{j}=\log (j)^{1+\eta}$, and let $s_{j}=$ $\left[j \log (j)^{-\kappa}\right]$. Now, for $j \geq 0$ and $n \geq 1$, on $\Omega \times[0,1)$ define the random variables

$$
\boldsymbol{U}^{(j)}(\omega, t)=\left\|\mu_{q}^{w^{(j)}(t)}(\omega)\right\|, \quad \boldsymbol{V}_{n}^{(j)}(\omega, t)=S_{n}^{N, \varepsilon_{n}, \eta_{n}}\left(\mu_{q}^{w^{(j)}(t)}(\omega), \mu^{w^{(j)}(t)}(\omega), \tau_{\mu}^{\prime}(q)\right) .
$$

To prove Theorem 3.3, by Proposition 2.2 we claim that it is enough to prove that

$$
\sum_{j \geq 0} \mathcal{Q}_{q}\left(\boldsymbol{U}^{(j)} \leq b^{-\rho_{j}}\right)<\infty \text { and } \sum_{j \geq 0} b^{\rho_{j} h} \mathrm{E}_{\mathcal{Q}_{q}}\left(\left(\sum_{n \geq \beta_{j}} \boldsymbol{V}_{n}^{(j)}\right)^{h}\right)<\infty
$$

for some $h \in(0,1]$, where $\mathrm{E}_{\mathcal{Q}_{q}}$ denotes expectation with respect to $\mathcal{Q}_{q}$. The main difference between the proofs of Proposition 2.2 and Theorem 3.2 is that here we do not seek a result valid uniformly over words $w$ of the same generation $j$, but only a result valid for $w^{(j)}(t)$, for $\mu_{q}$-almost every $t$. As a consequence, we must control only one pair of random variables $\left(\boldsymbol{U}^{(j)}, \boldsymbol{V}^{(j)}\right)$ in each generation, instead of $b^{j}$ random variables. This allows us to slow the rate of increase of $s_{j}$.

Fix an $h \in(0,1)$. Since $x \mapsto x^{h}$ is subadditive on $\mathbb{R}_{+}$, we have

$$
\mathrm{E}_{\mathcal{Q}_{q}}\left(\left(\sum_{n \geq s_{j}} \boldsymbol{V}_{n}^{(j)}\right)^{h}\right) \leq \sum_{n \geq s_{j}} \mathrm{E}_{\mathcal{Q}_{q}}\left(\left(\boldsymbol{V}_{n}^{(j)}\right)^{h}\right) .
$$

For $\omega \in \Omega^{*}, j \geq 1$, and $n \geq 1$, by definition of the measures $\mu_{q}$ and $\mu_{q}^{w}$ and since $\left(\mu_{q}^{w^{(j)}(t)}(\omega), \mu^{w^{(j)}(t)}(\omega)\right)$ does not depend on $t \in I_{w}$, we have

$$
\begin{aligned}
\int_{[0,1]}\left(\boldsymbol{V}_{n}^{(j)}(\omega, t)\right)^{h} \mu_{q}(\omega)(\mathrm{d} t) & =\sum_{w \in \mathcal{A}^{j}} \int_{I_{w}}\left(\boldsymbol{V}_{n}^{(j)}(\omega, t)\right)^{h} \mu_{q}(\omega)(\mathrm{d} t) \\
& =\sum_{w \in \mathcal{A}^{j}} \prod_{k=0}^{j-1} W_{q, w_{k+1}}(w \mid k) \int_{I_{w}}\left(\boldsymbol{V}_{n}^{(j)}(\omega, t)\right)^{h} \mu_{q}^{w}(\omega) \circ f_{I_{w}}^{-1}(\mathrm{~d} t) \\
& =\sum_{w \in \mathcal{A}^{j}}\left(\prod_{k=0}^{j-1} W_{q, w_{k+1}}(w \mid k)\right)\left(V_{n}^{w}\right)^{h}\left\|\mu_{q}^{w}\right\|
\end{aligned}
$$

where $V_{n}^{w}=S_{n}^{N, \varepsilon_{n}, \eta_{n}}\left(\mu_{q}^{w}(\omega), \mu^{w}(\omega), \tau_{\mu}^{\prime}(q)\right)$ is as defined in the proof of Theorem 3.2. The above sum is a random variable on $(\Omega, \mathscr{B}, \mathrm{P})$. In addition, in each of its terms, the product is independent of $\left(V_{n}^{w}\right)^{h}\left\|\mu_{q}^{w}\right\|$. Moreover, the probability distribution of $\left(V_{n}^{w}\right)^{h}\left\|\mu_{q}^{w}\right\|$ does not depend on $w$. Consequently, using the martingale property of the sequence $\left(\left\|\mu_{q, j}\right\|\right)_{j \geq 0}$, we obtain

$$
\mathrm{E}_{\mathcal{Q}_{q}}\left(\left(\boldsymbol{V}_{n}^{(j)}\right)^{h}\right)=\mathrm{E}\left(\left(V_{n}^{w}\right)^{h}\left\|\mu_{q}^{w}\right\|\right),
$$

where $w \in \mathscr{A}^{j}$. Let $p=1 /(1-h)$. The Hölder inequality yields

$$
\mathrm{E}\left(\left(V_{n}^{w}\right)^{h}\left\|\mu_{q}^{w}\right\|\right) \leq \mathrm{E}\left(V_{n}^{w}\right)^{h} \mathrm{E}\left(\left\|\mu_{q}\right\|^{p}\right)^{1 / p} .
$$

Finally, fix $p$ close enough to 1 that $\mathrm{E}\left(\left\|\mu_{q}\right\|^{p}\right)<\infty$ (see the proof of Lemma 4.3 for the existence of such a $p$ ). Then (4.4) implies that $\sum_{j \geq 1} b^{\rho_{j} h} \sum_{n \geq s_{j}}\left(\mathrm{E}\left(V_{n}^{w}\right)\right)^{h}<\infty$, and the result follows. 
Computations similar to those above show that, for every $j \geq 1$,

$$
\mathcal{Q}_{q}\left(\boldsymbol{U}^{(j)} \leq b^{-\rho_{j}}\right)=\mathrm{E}\left(\mathbf{1}_{\left\{Y_{q} \leq b^{\left.-\rho_{j}\right\}}\right.} Y_{q}\right) \leq b^{-\rho_{j}} \mathrm{P}\left(Y_{q} \leq b^{-\rho_{j}}\right) .
$$

It follows from Lemma 4.2.1 that, for some $h>0$, we have $\mathrm{P}\left(Y_{q} \leq x\right)=O\left(x^{h}\right)$ as $x \rightarrow 0$. This implies that $\sum_{j \geq 1} \mathcal{Q}_{q}\left(\boldsymbol{U}^{(j)} \leq b^{-\rho_{j}}\right)<\infty$.

We now prove part 2 of the theorem. The proof is similar to that of part 1 . It is enough to prove the result for a compact subinterval, $K$, of $\mathcal{O}$, instead of for $\mathcal{O}$ itself. Fix such an interval $K$. The idea is now to consider on $(K \times \Omega \times[0,1], \mathscr{B}(K) \otimes \mathscr{B} \otimes \mathscr{B}([0,1]))$ the probability distribution $\mathcal{Q}_{K}$ such that

$$
\mathcal{Q}_{K}(A)=\int_{K} \mathrm{E}_{\mathcal{Q}_{q}}\left(\mathbf{1}_{A}(q, \omega, t)\right) \frac{\mathrm{d} q}{|K|}, \quad A \in \mathscr{B}(K) \otimes \mathscr{B} \otimes \mathscr{B}([0,1]) .
$$

Then $\boldsymbol{U}^{(j)}(q, \omega, t)$ and $\boldsymbol{V}_{n}^{(j)}(q, \omega, t)$ are redefined as

$$
\begin{aligned}
& \boldsymbol{U}^{(j)}(q, \omega, t)=\left\|\mu_{q}^{w^{(j)}(t)}(\omega)\right\|, \\
& \boldsymbol{V}_{n}^{(j)}(q, \omega, t)=S_{n}^{N, \varepsilon_{n}, \eta_{n}}\left(\mu_{q}^{w^{(j)}(t)}(\omega), \mu^{w^{(j)}(t)}(\omega), \tau_{\mu}^{\prime}(q)\right) .
\end{aligned}
$$

Since there exists a $p>1$ such that $M=\sup _{q \in K} \mathrm{E}\left(\left\|\mu_{q}\right\|^{p}\right)^{1 / p}<\infty$ (again, see the proof of Lemma 4.3), the computations performed above yield

$$
\sum_{j \geq 0} b^{\rho_{j} h} \sum_{n \geq \gamma_{j}} \mathrm{E}_{\mathcal{Q}_{K}}\left(\left(\boldsymbol{V}_{n}^{(j)}\right)^{h}\right) \leq|K| M \sum_{j \geq 1} b^{\rho_{j} h} \sum_{n \geq \gamma_{j}}\left(\mathrm{E}\left(V_{n}^{w}\right)\right)^{h}<\infty .
$$

Finally, $\sum_{j \geq 0} \mathcal{Q}_{K}\left(\boldsymbol{U}^{(j)} \leq b^{-\rho_{j}}\right) \leq|K| \sum_{j \geq 1} b^{-\rho_{j}} \mathrm{P}\left(\inf _{q \in K} Y_{q} \leq b^{-\rho_{j}}\right)$, which is finite according to part 1 of Lemma 4.2.

\subsection{Proof of Theorem 3.4}

We assume without loss of generality that $K$ contains the point 1 , and we define $q_{K}=$ $\max \{|q|: q \in K\}$. Recall that, for $j \geq 0$ and $n \geq 1$, if $(w, v) \in \mathcal{A}^{j} \times \mathcal{A}^{n}$ and $q \in K$ then

$$
\mu^{w}\left(I_{v}\right)^{q}=\mu_{q}^{w}\left(I_{v}\right) b^{-n \tilde{\tau}_{\mu}(q)} \frac{Y(w v)^{q}}{Y_{q}(w v)} .
$$

Summing over $v \in \mathcal{A}^{n}$ yields

$$
\begin{aligned}
& Y_{q}(w) b^{-n \tilde{\tau}_{\mu}(q)} \inf _{q \in K, v \in \mathcal{A}^{n}} \frac{Y(w v)^{q}}{Y_{q}(w v)} \leq b^{-n \tau_{n}^{w}(q)}, \\
& b^{-n \tau_{n}^{w}(q)} \leq Y_{q}(w) b^{-n \tilde{\tau}_{\mu}(q)} \sup _{q \in K, v \in \mathcal{A}^{n}} \frac{Y(w v)^{q}}{Y_{q}(w v)} .
\end{aligned}
$$

Fix $\delta \in(0,1)$ and $\theta>0$ such that the conclusions of Propositions 4.1 and 4.2, below, hold. Then, with probability 1 , for large enough $j$, every $w \in \mathcal{A}^{j}, q \in K$, and $n \geq j^{\delta}$, we have $b^{-n \tau_{n}^{w}(q)} \leq Y_{q}(w) b^{-n \tilde{\tau}_{\mu}(q)} n^{\left(q_{K}+1\right) \theta}$. Now, remarking that

$$
\mathcal{N}_{n}\left(\mu^{w}, \tilde{\tau}_{\mu}^{\prime}(q), \varepsilon_{n}\right) \min \left(b^{-n q\left(\tilde{\tau}_{\mu}^{\prime}(q)+\varepsilon_{n}\right)}, b^{-n q\left(\tilde{\tau}_{\mu}^{\prime}(q)-\varepsilon_{n}\right)}\right) \leq b^{-n \tau_{n}^{w}(q)},
$$


we obtain

$$
\begin{aligned}
\mathcal{N}_{n}\left(\mu^{w}, \tilde{\tau}_{\mu}^{\prime}(q), \varepsilon_{n}\right) & \leq b^{n\left(\tilde{\tau}_{\mu}^{\prime}(q) q-\tau_{n}^{w}(q)+\operatorname{sgn}(q) q \varepsilon_{n}\right)} \\
& \leq Y_{q}(w) b^{n\left(\tilde{\tau}_{\mu}^{\prime}(q) q-\tilde{\tau}_{\mu}(q)+\operatorname{sgn}(q) q \varepsilon_{n}\right)} n^{\left(q_{K}+1\right) \theta} .
\end{aligned}
$$

On the other hand, owing to Theorem 3.2 and Proposition 4.1, there exists a $\theta>0$ such that, with probability 1 , for large enough $j$, all $w \in \mathcal{A}^{*}$, and $q \in K$, we have

$$
\mu_{q}^{w}\left(E_{\tilde{\tau}_{\mu}^{\prime}(q), s_{j}}^{\mu^{w}}(0, \tilde{\varepsilon}) \cap E_{\tilde{\tau}_{\mu}^{\prime}(q) q-\tilde{\tau}_{\mu}(q), s_{j}}^{\mu^{w}}(0, \tilde{\varepsilon})\right) \geq \frac{\left\|\mu_{q}^{w}\right\|}{2}=\frac{Y_{q}(w)}{2} .
$$

This implies that $b^{n\left(\tilde{\tau}_{\mu}^{\prime}(q) q-\tilde{\tau}_{\mu}(q)-\varepsilon_{n}\right)} Y_{q}(w) / 2 \leq \mathcal{N}_{n}\left(\mu^{w}, \tilde{\tau}_{\mu}^{\prime}(q), \varepsilon_{n}\right)$ for every $n \geq s_{j}$. Moreover, for large enough $j, j^{\delta} \leq s_{j}$. Thus, for large enough $n$,

$$
\sup _{q \in K} \operatorname{sgn}(q) q \varepsilon_{n}+\left(q_{K}+1\right) \theta \frac{\log _{b} n}{n}
$$

is controlled by $\left(1+q_{K}\right) \varepsilon_{n}$. The conclusion follows.

\subsection{Proof of Theorem 3.5}

We begin with three technical lemmas.

Lemma 4.3. Assume that $\mathcal{O}=\mathbb{R}$. For every compact subinterval $K$ of $\mathbb{R}$, there exist $C_{K}, c_{K}>$ 0 such that

$$
\sup _{q \in K} \mathrm{P}\left(Y_{q} \geq x\right) \leq C_{K} \exp \left(-c_{K} x\right) \text { for every } x \geq 1 .
$$

Proof. We recall the following properties involved in the proofs of several statements in Section 3. It is known (see [31] and [20]) that if $h>1$ then $\mathrm{E}\left(Y_{1}^{h}\right)<\infty$ if and only if $\mathrm{E}\left(\sum_{k=0}^{b-1} W_{k}^{h}\right)<1$. Consequently, if $q \in \mathcal{O}=\left\{q \in \mathbb{R}: \tilde{\tau}_{\mu}^{\prime}(q) q-\tilde{\tau}_{\mu}(q)>0\right\}$ and $h>1$, then $\mathrm{E}\left(Y_{q}^{h}\right)<\infty$ if and only if $\mathrm{E}\left(\sum_{k=0}^{b-1} W_{q, k}^{h}\right)<1$, that is, $\tilde{\tau}_{\mu}(q h)-h \tilde{\tau}_{\mu}(q)>0$. Moreover, it follows from Théorème VI.A.bi) of [4] that, for every compact subinterval $K$ of $\mathcal{O}$, there exists an $h>1$ such that $\sup _{q \in K} \mathrm{E}\left(Y_{q}^{h}\right)<\infty$.

That the mapping $q \mapsto \tilde{\tau}_{\mu}(q) / q$ is increasing on both $\mathbb{R}_{-}^{*}$ and $\mathbb{R}_{+}^{*}$ is equivalent to having $\mathcal{O}=\mathbb{R}$. As a consequence, we obtain $\tilde{\tau}_{\mu}(q h)-h \tilde{\tau}_{\mu}(q)>0$ for all $q \in \mathbb{R}$ and $h>1$, that is, $\mathrm{E}\left(Y_{q}^{h}\right)<\infty$. We also have $\left\|W_{q, k}\right\|_{\infty} \leq 1$ for all $q \in \mathbb{R}$ and $k, 0 \leq k \leq b-1$.

We fix $K$, a compact subset of $\mathbb{R}$, and consider the quantity defined by $t_{k}(q)=\mathrm{E}\left(Y_{q}^{k}\right) / k$ ! for $q \in K$ and $k \geq 1$, with $t_{0}(q)=1$. Using Equation (4.6) of [36] (our random variable $Y_{q}$ is denoted there by $W$ ), we find that, for every $k \geq 2$ and every $q \in K$,

$$
t_{k}(q) \leq c_{K} \sum_{\left\{\left(k_{0}, \ldots, k_{b-1}\right): 0 \leq k_{i} \leq k-1, k_{0}+\cdots+k_{b-1}=k\right\}} \prod_{i=0}^{b-1} t_{k_{i}}(q),
$$

where $c_{K}=\sup _{q \in K} \sup _{k \geq 2}\left(1-b^{-\tilde{\tau}_{\mu}(k q)+k \tilde{\tau}_{\mu}(q)}\right)^{-1}$. We see that

$$
c_{K}=\sup _{q \in K}\left(1-b^{-\tilde{\tau}_{\mu}(2 q)+2 \tilde{\tau}_{\mu}(q)}\right)^{-1}<\infty .
$$

Hence, with $\tilde{t}_{k}=\sup _{q \in K} t_{k}(q)$, we have

$$
\tilde{t}_{k} \leq c_{K} \sum_{\left\{\left(k_{0}, \ldots, k_{b-1}\right): 0 \leq k_{i} \leq k-1, k_{0}+\cdots+k_{b-1}=k\right\}} \prod_{i=0}^{b-1} \tilde{t}_{k_{i}} \text { for all } k \geq 2 .
$$


Since $\tilde{t}_{0}=\tilde{t}_{1}=1$, Lemma 2.6 of [25] yields $\lim \sup _{k \rightarrow \infty} \tilde{t}_{k}^{1 / k}<\infty$. This implies the existence of a constant $C>0$ such that

$$
\sup _{q \in K} \mathrm{E}\left(Y_{q}^{k}\right) \leq C^{k} k ! \quad \text { for all } k \geq 1 .
$$

Now fix $c_{K} \in\left(0, C^{-1}\right)$. For $x>0$, we have

$$
\sup _{q \in K} \mathrm{P}\left(Y_{q} \geq x\right) \leq \mathrm{e}^{-c_{K} x} \sup _{q \in K} \mathrm{E}\left(\mathrm{e}^{c_{K} Y_{q}}\right) \leq \mathrm{e}^{-c_{K} x} \sum_{k=0}^{\infty} \frac{c_{K}^{k}}{k !} \sup _{q \in K} \mathrm{E}\left(Y_{q}^{k}\right) \leq\left(1-c_{K} C\right)^{-1} \mathrm{e}^{-c_{K} x} .
$$

Remark 4.2. We are not able to control $\mathrm{P}\left(\sup _{q \in K} Y_{q} \geq x\right)$ at $\infty$. This is the reason why the next two lemmas are needed to obtain Proposition 4.2.

For $n \geq 1$, let $Q_{n}$ be the set of dyadic numbers of generation $n$.

Lemma 4.4. Let $K$ be a compact subinterval of $\mathcal{O}$, and let $\eta>0$. There exist $\chi \in(0,1)$ and $\delta \in(0,1)$ such that, with probability 1 ,

1. for large enough $j$, all $w \in \mathcal{A}^{j}$, all $n \geq\left[j^{1+\eta}\right]$, and all $q, q^{\prime} \in Q_{n}$ such that $\left|q-q^{\prime}\right|=$ $2^{-n}$, we have $\left|Y_{q}^{w}-Y_{q^{\prime}}^{w}\right| \leq\left|q^{\prime}-q\right|^{\chi}$; and

2. for large enough $j$, all $n \geq j^{\delta}$, all $w \in \mathcal{A}^{j}$, all $v \in \mathcal{A}^{n}$, all $m \geq\left[n^{1+\eta}\right]$, and all $q, q^{\prime} \in Q_{m}$ such that $\left|q^{\prime}-q\right|=2^{-m}$, we have $\left|Y_{q}^{w v}-Y_{q^{\prime}}^{w v}\right| \leq\left|q^{\prime}-q\right|^{\chi}$.

Proof. By Théorème VI.A.bi) of [4], there exist $h>1$ and $C_{K}>0$ such that

$$
\mathrm{E}\left(\left|Y_{q}-Y_{q^{\prime}}\right|^{h}\right) \leq C_{K}\left|q-q^{\prime}\right|^{h} \quad \text { for all }\left(q, q^{\prime}\right) \in K^{2} .
$$

For $n \geq 1$, let $\tilde{Q}_{n}$ be the set of pairs $\left(q, q^{\prime}\right) \in Q_{n}$ such that $\left|q-q^{\prime}\right|=2^{-n}$, and let $\chi \in$ $(0,(h-1) / h)$. Using (4.7) and the Markov inequality, we obtain

$$
\begin{aligned}
p_{n} & :=\mathrm{P}\left(\text { there exists }\left(q, q^{\prime}\right) \in \tilde{Q}_{n} \text { such that }\left|Y_{q}-Y_{q^{\prime}}\right| \geq\left|q-q^{\prime}\right|^{\chi}\right) \\
& \leq \sum_{\left(q, q^{\prime}\right) \in \tilde{Q}_{n}} \mathrm{P}\left(\left|Y_{q}-Y_{q^{\prime}}\right| \geq\left|q-q^{\prime}\right|^{\chi}\right) \\
& \leq 2|K| 2^{n} C_{K} 2^{n \times h} 2^{-n h} .
\end{aligned}
$$

Fix $\eta>0$ and $\delta \in\left((1+\eta)^{-1}, 1\right)$. The inequality $\sum_{j \geq 1} b^{j} \sum_{n \geq\left[j^{1+\eta}\right]} p_{n}<\infty$ implies part 1 of the lemma, by the Borel-Cantelli lemma. Part 2 follows from the fact that

$$
\sum_{j \geq 1} b^{j} \sum_{n \geq j^{\delta}} b^{n} \sum_{m \geq\left[n^{1+\eta}\right]} p_{m}<\infty .
$$

Lemma 4.5. Under the assumptions of Theorem 3.4, let $K \subset \mathbb{R}$ be a compact interval. Let $\eta>0$. There exist $\delta \in(0,1)$ and $\theta>1$ such that, with probability 1 ,

1. for large enough $j$ and all $w \in \mathcal{A}^{j}$, we have $\sup _{q \in Q_{[j 1+\eta]} \cap K} Y_{q}(w) \leq j^{\theta}$; and

2. for large enough $j$, all $n \geq j^{\delta}$, and all $(v, w) \in \mathcal{A}^{n} \times \mathcal{A}^{j}$, we have

$$
\sup _{q \in Q_{\left[n^{1+\eta}\right]} \cap K} Y_{q}(w v) \leq n^{\theta} .
$$


Proof. Fix $\theta>1+\eta$. For $q \in K$ and $j \geq 1$, define $p_{j}(q)=\mathrm{P}\left(Y_{q} \geq j^{\theta}\right)$. By Lemma 4.3, for all $j \geq 1$ we have

$$
\mathrm{P}\left(\sup _{q \in Q_{[j}^{1+\eta]} \cap K} Y_{q} \geq j^{\theta}\right) \leq \sum_{q \in Q_{[j}^{1+\eta]} \cap K} p_{j}(q) \leq p_{j}:=2 C_{K}|K| 2^{j^{1+\eta}} \exp \left(-c_{K} j^{\theta_{K}}\right) .
$$

We leave it to the reader to verify that $\sum_{j \geq 1} b^{j} p_{j}<\infty$ and $\sum_{j \geq 1} b^{j} \sum_{n \geq j^{\delta}} b^{n} p_{n}<\infty$ if $\delta \in\left(\theta_{K}^{-1}, 1\right)$. This yields both parts of Lemma 4.5 .

The following two propositions are needed to control the inequality (4.6).

Proposition 4.1. Under the assumptions of Theorem 3.2, let $K$ be a compact subinterval of $\mathcal{O} \cap \mathbb{R}_{+}$or $\mathcal{O} \cap \mathbb{R}_{-}$. There exist $\theta>0$ and $\delta \in(0,1)$ such that, with probability 1 ,

1. for large enough $j$, and all $w \in \mathcal{A}^{j}$, we have $\inf _{q \in K} Y_{q}(w) \geq j^{-\theta}$; and

2. for large enough $j$, all $n \geq j^{\delta}$, all $w \in \mathcal{A}^{j}$, and all $v \in \mathcal{A}^{n}$, we have

$$
\inf _{q \in K} Y_{q}(w v) \geq n^{-\theta} \text {. }
$$

Proof. Fix a $\theta>1$ such that $\theta_{K}=\theta \gamma_{K} /\left(1-\gamma_{K}\right)>1$, where $\gamma_{K}$ is as in Lemma 4.2. Also define $p_{j}:=\mathrm{P}\left(\inf _{q \in K} Y_{q}<j^{-\theta}\right)$. We leave it to the reader to verify, using Lemma 4.2, that $\sum_{j \geq 1} b^{j} p_{j}<\infty$ and that if $\delta \in\left(\theta_{K}^{-1}, 1\right)$ then $\sum_{j \geq 1} b^{j} \sum_{n \geq j^{\delta}} b^{n} p_{n}<\infty$. This yields both parts of the proposition.

Proposition 4.2. Under the assumptions of Theorem 3.4, let $K \subset \mathcal{O}$ be a compact interval. There exist $\theta>0$ and $\delta \in(0,1)$ such that, with probability 1 ,

1. for large enough $j$ and all $w \in \mathcal{A}^{j}$, we have $\sup _{q \in K} Y_{q}(w) \leq j^{\theta}$; and

2. for large enough $j$, all $n \geq j^{\delta}$, all $w \in \mathcal{A}^{j}$, and all $v \in \mathcal{A}^{n}$, we have

$$
\sup _{q \in K} Y_{q}(w v) \leq n^{\theta} .
$$

Proof. We assume without loss of generality that the endpoints of $K$ are dyadic numbers. It is well known (see the proof of Kolmogorov's theorem in [32, pp. 53-55]) that Lemma 4.4 implies the existence of a constant $C_{K}>0$ such that, with probability 1 ,

1. for large enough $j$, all $w \in \mathcal{A}^{j}$, and all $q, q^{\prime} \in K$ such that $\left|q-q^{\prime}\right| \leq 2^{-\left[j^{1+\eta}\right]}$, we have $\left|Y_{q}(w)-Y_{q^{\prime}}(w)\right| \leq C_{K}\left|q^{\prime}-q\right|^{\chi} ;$ and

2. for large enough $j$, all $n \geq j^{\delta}$, all $(v, w) \in \mathcal{A}^{n} \times \mathcal{A}^{j}$, and all $q, q^{\prime} \in K$ such that $\left|q^{\prime}-q\right| \leq 2^{-\left[n^{1+\eta}\right]}$, we have $\left|Y_{q}(w v)-Y_{q^{\prime}}(w v)\right| \leq C_{K}\left|q^{\prime}-q\right|^{\chi}$.

The result then follows from Lemma 4.5.

Finally, Theorem 3.5 is a consequence of (4.6) and Propositions 4.1 and 4.2.

\section{Growth speed as a tool for conditioned ubiquity results}

Let $\left\{\left(x_{n}, \lambda_{n}\right)\right\}_{n \geq 1}$ be a sequence in $[0,1] \times(0,1]$ such that $\lim _{n \rightarrow \infty} \lambda_{n}=0$. For every $t \in(0,1), k \geq 1$, and $r \in(0,1)$, we consider the set of balls

$$
\mathcal{B}_{k, r}(t)=\left\{B\left(x_{n}, \lambda_{n}\right): t \in B\left(x_{n}, r \lambda_{n}\right), \lambda_{n} \in\left(b^{-(k+1)}, b^{-k}\right]\right\}
$$


( $B\left(y, r^{\prime}\right)$ is the closed interval centered at $y$ with radius $\left.r^{\prime}\right)$. Note that this set may be empty. Then, if $\xi>1$ and $B\left(x_{n}, \lambda_{n}\right) \in \mathcal{B}_{k, 1 / 2}(t)$, let $\mathcal{B}_{k}^{\xi}(t)$ be the set of $b$-adic intervals of maximal length included in $B\left(x_{n}, \lambda_{n}^{\xi}\right)$.

The next result is key in building a generalized Cantor set of Hausdorff dimension greater than or equal to $\tau_{\mu}^{*}(h) / \xi$ in the set $K(\mu, h, \xi, \tilde{x}, \tilde{\lambda}, \tilde{\varepsilon}$ ) (see (1.6)), when $\mu$ is a Mandelbrot measure. In the construction of this Cantor set, the following property related to the control of the growth speed is used repeatedly: if $q \in \mathcal{O}$ and $h=\tau_{\mu}^{\prime}(q)$, then each measure $\mu_{q}^{u}$ is carried by the set $E_{\tau_{\mu}^{*}(h)}^{\mu_{u}^{u}}(N, \tilde{\varepsilon})$ and, roughly speaking, if $G S\left(\mu_{q}^{u}, \mu_{q}^{u}, \tau_{\mu}^{*}(h), N, \tilde{\varepsilon}\right)$ is not too large, the measure $\mu_{q}^{u}$ restricted to $E_{\tau_{\mu}^{*}(h)}^{\mu_{u}^{u}}(N, \tilde{\varepsilon})$ can be viewed as being monofractal of exponent $\tau_{\mu}^{*}(h)$.

Theorem 5.1. Suppose that $\lim _{\sup _{n \rightarrow \infty}} B\left(x_{n}, \lambda_{n} / 4\right)=(0,1)$, let $\mu$ be an independent random cascade, and fix a $\kappa>0$. For $j \geq 2$, let $\S_{j}=j \log (j)^{-\kappa}$ and $\rho_{j}=\log (j)^{\chi}$ with $\chi>1$. Assume that (3.4) holds.

For every $q \in \mathcal{O}$ and $\xi>1$, with probability 1 the following property (property $\mathcal{P}(\xi, q)$ ) holds: for $\mu_{q}$-almost every $t$, there are infinitely many $k \geq 1$ such that $\mathscr{B}_{k, 1 / 2}(t) \neq \varnothing$, and there exists a $u \in\left\{v \in \mathcal{A}^{*}:\right.$ there exists an $I \in \mathscr{B}_{k}^{\xi}(t)$ with $\left.I=I_{v}\right\}$ such that

$$
G S\left(\mu_{q}^{u}, \mu_{q}^{u}, \tau_{\mu}^{\prime}(q) q-\tau_{\mu}(q), N, \tilde{\varepsilon}\right) \leq \diamond_{|u|} \quad \text { and }\left\|\mu_{q}^{u}\right\| \geq b^{-\rho_{|u|}} .
$$

Remark 5.1. 1. Under the assumptions of Theorem 5.1, Theorems 3.1 and 5.1, which are associated with the main result on heterogeneous ubiquity established in [8], imply that, for every $q \in \mathcal{O}$ and $\xi>1$, with probability 1 ,

$$
\operatorname{dim} K\left(\mu, \tau_{\mu}^{\prime}(q), \xi, \tilde{x}, \tilde{\lambda}, \tilde{\varepsilon}\right) \geq \frac{\tau_{\mu}^{\prime}(q) q-\tau_{\mu}(q)}{\xi} .
$$

2. If $q$ is fixed in $\mathcal{O}$ then the assumption that $\lim _{\sup } \operatorname{sum}_{n \rightarrow \infty} B\left(x_{n}, \lambda_{n} / 4\right)=(0,1)$ can be weakened by instead requiring only that $\lim _{n \rightarrow \infty} B\left(x_{n}, \lambda_{n} / 4\right)$ is of full $\mu_{q}$-measure.

3. The result of [10] on ubiquity conditioned by Mandelbrot measures concerns the case where $\left\{\left(x_{n}, \lambda_{n}\right)\right\}_{n}=\left\{\left(k b^{-j}, b^{-j}\right)\right\}_{j \geq 1,0 \leq k<b^{-j}}$. There a slightly different version of $\mathcal{P}(\xi, q)$ is invoked whose proof is easily deduced from that of Theorem 5.1.

Proof of Theorem 5.1. For $k \geq 1$ and $w \in \mathcal{A}^{k+3}$, note that $\mathcal{B}_{k, 1 / 4}(t) \subset \mathcal{B}_{k, 1 / 2}(s)$ for all $t, s \in I_{w}$. Let $\mathcal{R}_{w}=\left\{n\right.$ : there exists a $t \in I_{w}$ such that $\left.B\left(x_{n}, \lambda_{n}\right) \in \mathcal{B}_{k, 1 / 4}(t)\right\}$. Define $n(w)=\inf \left\{n: x_{n}=\min \left\{x_{m}: m \in \mathcal{R}_{w}\right\}\right\}$ if $\mathcal{R}_{w} \neq \varnothing$ and $n(w)=0$ otherwise.

If $\xi>1$ and $n(w)>0$, then let $u(w)$ be the word that encodes the $b$-adic interval of maximal length included in $B\left(x_{n}, \lambda_{n}^{\xi}\right)$ and whose left-hand endpoint is minimal. If $\xi>1$ and $n(w)=0$, then let $u(w)$ be the word of generation $[\xi|w|]$ that has prefix $w$ and whose [ $\xi|w|]-|w|$ last digits equal 0 .

Now, $w^{(j)}(t)$ being as defined in the statement of Theorem 3.3, we prove a slightly stronger result than Theorem 5.1. For every $q \in \mathcal{O}$ and $\xi>1$, with probability 1 the following property (property $\tilde{\mathcal{P}}(\xi, q)$ ) holds: for $\mu_{q}$-almost every $t$, if $j$ is large enough then, for all $k \geq j$ such that $n\left(w_{k+3}(t)\right)>0, u \equiv u\left(w_{k+3}(t)\right)$ satisfies (5.1). In the sequel we denote $u\left(w_{k+3}(t)\right)$ by $u_{k, \xi}(t)$.

Fix $\xi>1$ and $q \in K$. For $j \geq 0$ and $n \geq 1$, define on $\Omega \times[0,1)$ the random variables

$$
\begin{aligned}
& \boldsymbol{U}^{(j)}(\omega, t)=\left\|\mu_{q}^{u_{j, \xi}(t)}(\omega)\right\|, \\
& \boldsymbol{V}_{n}^{(j)}(\omega, t)=S_{n}^{N, \varepsilon_{n}, \eta_{n}}\left(\mu_{q}^{u_{j, \xi}(t)}(\omega), \mu_{q}^{u_{j, \xi}(t)}(\omega), q \tau_{\mu}^{\prime}(q)-\tau_{\mu}^{\prime}(q)\right) .
\end{aligned}
$$


We can use the proof of Proposition 2.2 to deduce that it is enough to prove that

$$
\begin{array}{r}
\sum_{j \geq 1} \mathcal{Q}_{q}\left(\left\{\text { there exists a } k \geq j \text { such that } b^{\rho_{\left|u_{k, \xi}(t)\right|}} \sum_{n \geq s_{\left|u_{k, \xi}(t)\right|}} V_{n}^{(k)}(\omega, t) \geq \frac{1}{2}\right\}\right)<\infty, \\
\sum_{j \geq 1} \mathcal{Q}_{q}\left(\left\{\text { there exists a } k \geq j \text { such that } \boldsymbol{U}^{(j)}(\omega, t) \leq b^{\left.\left.-\rho_{\left|u_{k, \xi}(t)\right|}\right\}\right)<\infty .}\right.\right.
\end{array}
$$

Since there exist $c$ and $c^{\prime}, c>c^{\prime}>0$, such that $c^{\prime} \xi k \leq\left|u_{k, \xi}(t)\right| \leq c \xi k$ for all $t$, in order to prove (5.2) and (5.3) it is enough to show that

$$
\begin{aligned}
\mathcal{T} & :=\sum_{j \geq 1} \sum_{k \geq j} \mathcal{Q}_{q}\left(b^{\rho_{\bar{k}}} \sum_{n \geq \delta_{\tilde{k}}} \boldsymbol{V}_{n}^{(k)}(\omega, t) \geq \frac{1}{2}\right)<\infty, \\
\mathcal{T}^{\prime} & :=\sum_{j \geq 1} \sum_{k \geq j} \mathcal{Q}_{q}\left(\boldsymbol{U}^{(k)} \leq b^{-\rho_{\tilde{k}}}\right)<\infty,
\end{aligned}
$$

where $\bar{k}=[c \xi k]+1$ and $\tilde{k}=\left[c^{\prime} \xi k\right]$. Note that

$$
\mathcal{T} \leq 2^{h} \sum_{j \geq 1} \sum_{k \geq j} \sum_{n \geq \delta_{\tilde{k}}} b^{\rho_{\bar{k}}^{h}} \mathrm{E}_{\mathcal{Q}_{q}}\left(\left(\boldsymbol{V}_{n}^{(k)}\right)^{h}\right)
$$

if $h \in(0,1)$. Mimicking the computations performed in the proof of Theorem 3.3, we obtain

$$
\int_{[0,1]}\left(V_{n}^{u_{k, \xi}(t)}(\omega)\right)^{h} \mu_{q}(\omega)(\mathrm{d} t)=\sum_{w \in \mathcal{A}^{k+3}}\left(\prod_{k=0}^{k-1} W_{q, w_{k+1}}(w \mid k)\right)\left(V_{n}^{u(w)}\right)^{h}\left\|\mu_{q}^{w}\right\| .
$$

Using the independence of the random variables as well as $p$ and $h$ as in the proof of Theorem 3.3, we obtain

$$
\mathrm{E}_{\mathcal{Q}_{q}}\left(\left(\boldsymbol{V}_{n}^{(k)}\right)^{h}\right) \leq \mathrm{E}\left(V_{n}^{u(w)}\right)^{h} \mathrm{E}\left(\left\|\mu_{q}^{w}\right\|^{p}\right)^{1 / p},
$$

where $w$ is any element of $\mathcal{A}^{*}$. Then our choices for $\rho_{j}$ and $\delta_{j}$ ensure that $\mathcal{T}$ is finite.

For any $h^{\prime}>0, \mathcal{T}^{\prime} \leq \sum_{j \geq 1} \sum_{k \geq j} b^{-\rho_{\tilde{k}} h} \mathrm{E}_{\mathcal{Q}_{q}}\left(\left(\boldsymbol{U}^{(k)}\right)^{-h^{\prime}}\right)$. A computation analogous to the one above shows that, with the same $h$ and $p$,

$$
\mathrm{E}_{\mathcal{Q}_{q}}\left(\left(\boldsymbol{U}^{(k)}\right)^{-h^{\prime}}\right) \leq\left(\mathrm{E}\left(Y_{q}^{u(w)}\right)^{-h^{\prime} / h}\right)^{h} \mathrm{E}\left(\left\|\mu_{q}^{w}\right\|^{p}\right)^{1 / p}
$$

for any element $w$ of $\mathcal{A}^{*}$. If $h^{\prime}$ is small enough, then the right-hand side is bounded independently of $k$ according to Lemma 4.2, and the conclusion follows from our choice for $\rho_{j}$.

\section{Acknowledgement}

The authors are grateful to the referee for valuable comments.

\section{References}

[1] Arbeiter, M. and Patzschke, N. (1996). Random self-similar multifractals. Math. Nachr. 181, 5-42.

[2] Arnold, V. I. (1963). Small denominators and problems of stability of motion in classical and celestial mechanics. Ups. Mat. Nauk. 18, 91-192 (in Russian). English translation: Russian Math. Surveys 18, 85-191.

[3] Bacry, E. ANd Muzy, J.-F. (2003). Log-infinitely divisible multifractal processes. Commun. Math. Phys. 236, 449-475. 
[4] Barral, J. (1999). Moments, continuité et analyse multifractale des martingales de Mandelbrot. Prob. Theory Relat. Fields 113, 535-569.

[5] Barral, J. (2000). Continuity of the multifractal spectrum of a random statistically self-similar measure. J. Theoret. Prob. 13, 1027-1060.

[6] Barral, J. and Mandelbrot, B. B. (2002). Multifractal products of cylindrical pulses. Prob. Theory Relat. Fields 124, 409-430.

[7] Barral, J. And Mandelbrot, B. B. (2004). Random multiplicative multifractal measures. In Fractal Geometry and Applications, eds M. L. Lapidus and M. van Frankenhuijsen, AMS, Providence, RI, pp. 3-90.

[8] Barral, J. and Seuret, S. (2004). Heterogeneous ubiquitous systems and Hausdorff dimension in $\mathbb{R}^{d}$. Submitted.

[9] Barral, J. and Seuret, S. (2004). Sums of Dirac masses and conditioned ubiquity. C. R. Acad. Sci. Paris I 339, 787-792.

[10] Barral, J. AND Seuret, S. (2005). Combining multifractal additive and multiplicative chaos. Commun. Math. Phys. 257, 473-497.

[11] BARRAL, J. AND SEURET, S. (2005). The singularity spectrum of Lévy processes in multifractal time. To appear in Adv. Math.

[12] Barral, J. and Seuret, S. (2005). Inside singularities sets of random Gibbs measures. J. Statist. Phys. 120, 1101-1124.

[13] Barral, J., Ben Nasr, F. And Peyrière, J. (2003). Comparing multifractal formalisms: the neighboring boxes conditions. Asian J. Math. 7, 149-165.

[14] Barral, J., Coppens, M. O. and Mandelbrot, B. B. (2003). Multiperiodic multifractal martingale measures. J. Math. Pure Appl. 82, 1555-1589.

[15] Biggins, J. D. (1992). Uniform convergence of martingales in the branching random walk. Ann. Prob. 20, 137-151.

[16] Billingsley, P. (1965). Ergodic Theory and Information. John Wiley, New York.

[17] Brown, G., Michon, G. And Peyrière, J. (1992). On the multifractal analysis of measures. J. Statist. Phys. 66, 775-790.

[18] Collet, P. And Koukiou, F. (1992). Large deviations for multiplicative chaos. Commun. Math. Phys. 147, 329-342.

[19] Dodson, M. M. (2002). Exceptional sets in dynamical systems and Diophantine approximation. In Rigidity in Dynamics and Geometry, Springer, Berlin, pp. 77-98.

[20] Durrett, R. And Liggett, T. (1983). Fixed points of the smoothing transformation. Z. Wahrscheinlichkeitsth. 64, 275-301.

[21] Falconer, K. J. (1994). The multifractal spectrum of statistically self-similar measures. J. Theoret. Prob. 7, 681-702.

[22] FALConer, K. J. (2000). Representation of families of sets by measures, dimension spectra and Diophantine approximation. Math. Proc. Camb. Phil. Soc. 128, 111-121.

[23] Fan, A. H. (1997). Multifractal analysis of infinite products. J. Statist. Phys. 86, 1313-1336.

[24] Frisch, U. and Parisi, G. (1985). Fully developed turbulence and intermittency. In Proc. Internat. Summer School Phys., North-Holland, Amsterdam, pp. 84-88.

[25] Graf, S., Mauldin, R. D. and Williams, S. C. (1988). The exact Hausdorff dimension in random recursive constructions. Mem. Amer. Math. Soc. 71, 381.

[26] Halsey, T. C. et al. (1986). Fractal measures and their singularities: the characterization of strange sets. Phys. Rev. A 33, 1141-1151. (Correction: 34 (1986), 1601.)

[27] Holley, R. and Waymire, E. C. (1992). Multifractal dimensions and scaling exponents for strongly bounded random fractals. Ann. Appl. Prob. 2, 819-845.

[28] Jaffard, S. (1999). The multifractal nature of Lévy processes. Prob. Theory Relat. Fields 114, $207-227$.

[29] Kahane, J.-P. (1985). Sur le chaos multiplicatif. Ann. Sci. Math. Québec 9, 105-150.

[30] Kahane, J.-P. (1987). Positive martingales and random measures. Chinese Ann. Math. B 8, 1-12.

[31] Kahane, J.-P. And Peyrière, J. (1976). Sur certaines martingales de Benoît Mandelbrot. Adv. Math. 22, 131-145.

[32] Karatzas, I. And Shreve, S. E. (1988). Brownian Motion and Stochastic Calculus. Springer, New York.

[33] Khanin, K. And Kifer, Y. (1996). Thermodynamic formalism for random transformations and statistical mechanics. Amer. Math. Soc. Transl. 171, 107-140.

[34] KIFER, Y. (1995). Fractals via random iterated function systems and random geometric constructions. In Fractal Geometry and Stochastics (Finsterbergen, 1994; Progress Prob. 37), Birkhäuser, Basel, pp. 145-164.

[35] LÉVy VÉHel, J. AND Riedi, R. H. (1997). TCP traffic is multifractal: a numerical study. Res. Rep. RR-3129, INRIA.

[36] LiU, Q. (1996). The growth of an entire characteristic function and the tail probabilities of the limit of a tree martingale. In Trees (Progress Prob. 40), Birkhäuser, Basel, pp. 51-80. 
[37] Liu, Q. (2000). On generalized multiplicative cascades. Stoch. Process. Appl. 86, 263-286.

[38] LiU, Q. (2001). Asymptotic properties and absolute continuity of laws stable by random weighted mean. Stoch. Process. Appl. 95, 83-107.

[39] Mandelbrot, B. B. (1974). Intermittent turbulence in self-similar cascades: divergence of high moments and dimension of the carrier. J. Fluid Mech. 62, 331-358.

[40] Mandelbrot, B. B. (1997). Fractals and Scaling in Finance. Discontinuity, Concentration, Risk. Springer, New York.

[41] Mandelbrot, B. B. (2001). Scaling in financial prices. III. Cartoon Brownian motions in multifractal time. Quant. Finance 1, 427-440.

[42] Mandelbrot, B. B., Fisher, L. and Calvet, A. (1997). A multifractal model for asset returns. CFDP 1164. Available at http://cowles.econ.yale.edu/P/cd/dy1997.htm.

[43] Molchan, G. M. (1996). Scaling exponents and multifractal dimensions for independent random cascades. Commun. Math. Phys. 179, 681-702.

[44] MuZY, J.-F. AND BACRY, E. (2002). Multifractal stationary random measures and multifractal random walks with log infinitely divisible scaling laws. Phys. Rev. E 66, 056121.

[45] Olsen, L. (1994). Random Geometrically Graph Directed Self-similar Multifractals (Pitman Res. Notes Math. Ser. 307). John Wiley, New York.

[46] Olsen, L. (1995). A multifractal formalism. Adv. Math. 116, 82-196.

[47] Ossiander, M. And Waymire, E. C. (2000). Statistical estimation for multiplicative cascades. Ann. Statist. 28, 1533-1560.

[48] Parry, W. And Pollicott, M. (1990). Zeta Functions and the Periodic Orbit Structure of Hyperbolic Dynamics (Astérisque 187-188). Société Mathématique de France, Paris.

[49] RIEDI, R. (1995). An improved multifractal formalism and self-similar measures. J. Math. Anal. Appl. 189, 462-490.

[50] RIEDI, R. (2003). Multifractal processes. In Theory and Applications of Long-Range Dependence, Birkhäuser, Boston, MA, pp. 625-716. 